University of Nebraska - Lincoln

DigitalCommons@University of Nebraska - Lincoln

Papers in Solid Phase Dynamics

Chemical and Biomolecular Engineering

Research and Publications

$1-23-2002$

\title{
Solitary waves and supersonic reaction front in metastable solids
}

Hendrik J. Viljoen

University of Nebraska-Lincoln, hviljoen1@unl.edu

Lee L. Lauderback

Deparment of Chemical and Biomolecular Engineering, University of Nebraska Lincoln-68588,

llauderback1@unlserve.unl.edu

Didier Sornette

Institute of Geophysics and Planetary Sciences and Department of Earth and Space Sciences, University of California, Los Angeles, California, 90095

Follow this and additional works at: https://digitalcommons.unl.edu/chemengsolidphase

Part of the Chemical Engineering Commons

Viljoen, Hendrik J.; Lauderback, Lee L.; and Sornette, Didier, "Solitary waves and supersonic reaction front in metastable solids" (2002). Papers in Solid Phase Dynamics. 1.

https://digitalcommons.unl.edu/chemengsolidphase/1

This Article is brought to you for free and open access by the Chemical and Biomolecular Engineering Research and Publications at DigitalCommons@University of Nebraska - Lincoln. It has been accepted for inclusion in Papers in Solid Phase Dynamics by an authorized administrator of DigitalCommons@University of Nebraska - Lincoln. 


\title{
Solitary waves and supersonic reaction front in metastable solids
}

\author{
Hendrik J. Viljoen and Lee L. Lauderback \\ Department of Chemical Engineering, University of Nebraska-Lincoln, Lincoln, Nebraska 68588-0126
}

Didier Sornette

Institute of Geophysics and Planetary Sciences and Department of Earth and Space Sciences, University of California, Los Angeles, California 90095

and Laboratoire de Physique de la Matiere Condensee, CNRS UMR6622, and Universite des Sciences, Boite Postale 70, Parc Valrose, 06108 Nice Cedex 2, France

(Received 15 May 2001; published 23 January 2002)

\begin{abstract}
Motivated by an increasing number of remarkable experimental observations on the role of pressure and shear stress in solid reactions, explosions, and detonations, we present a simple one-dimensional model that embodies nonlinear elasticity and dispersion as well as chemical or phase transformation. This generalization of the Toda lattice provides an effective model for the description of the organization during an abrupt transformation in a solid. One of the challenges is to capture both the equilibrium degrees of freedom as well as to quantify the possible role of out-of-equilibrium perturbations. In the Toda lattice, we verify that the particle velocities converge in distribution towards the Maxwell-Boltzmann distribution, thus allowing us to define a bonafide temperature. In addition, the balance between nonlinearity and wave dispersion may create solitary waves that act as energy traps. In the presence of reactive chemistry, we show that the trapping of the released chemical energy in solitary waves that are excited by an initial perturbation provides a positive feedback that enhances the reaction rate and leads to supersonic explosion front propagation. These modes of rupture observed in our model may provide a first-order description of ultrafast reactions of heterogeneous mixtures under mechanical loading.
\end{abstract}

DOI: 10.1103/PhysRevE.65.026609

PACS number(s): 43.25.+y, 81.40.Np, 62.50.+p

\section{EXPERIMENTAL MOTIVATIONS}

Diffusion transfers of mass or heat usually control front propagation associated with solid phase chemical reactions or phase transformations. As a consequence, the velocity of fronts is small and even negligible compared to the sound velocities of the reactants and of the products. Typical solidsolid reactions such as $\mathrm{Ta}+\mathrm{C} \rightarrow \mathrm{TaC}$ or solid-liquid reactions such as $2 \mathrm{Al}+\mathrm{Fe}_{2} \mathrm{O}_{3} \rightarrow \mathrm{Al}_{2} \mathrm{O}_{3}+2 \mathrm{Fe}$, characterized by extremely high activation energies, can react in the combustion mode and these rates are determined by the preheating of reactants by thermal conduction. The combustion front velocity is proportional to $\sqrt{\kappa / \tau}$, where the thermal diffusivity

$$
\kappa=\frac{k}{\rho C_{p}} \approx \frac{O\left(10^{1}\right)}{O\left(10^{3}\right) \times O\left(10^{3}\right)} \approx O\left(10^{-6}\right),
$$

and the characteristic reaction time $\tau=1 / k_{0} e^{-E / R T_{\mathrm{ad}}}$ $\approx O\left(10^{-2}\right)$. Therefore, the reaction front velocity is of the order of $\nu \propto \sqrt{\kappa / \tau} \approx O\left(10^{-2}\right) \mathrm{m} / \mathrm{s}$. Thus, diffusive transfer cannot explain events propagating at front velocities much faster than $\mathrm{cm} / \mathrm{s}$, such as detonations or deflagrations, explosive recrystallization, photoinduced reactions, and the highpressure heterogeneous reactions studied by Bridgman in his pioneering work and later by Enikolopyan.

The ultrafast reaction of heterogeneous mixtures under mechanical loading is particularly intriguing. In 1935, Bridgman reported results of combined hydrostatic pressure and shear for a wide variety of materials [1]. Whilst most substances underwent polymorphic transformation, some reacted rather violently. In contrast to $\mathrm{PbO}$, that decomposed quiescently to a thin film of lead, $\mathrm{PbO}_{2}$ detonated and residue of $\mathrm{Pb}$ was found afterwards. Reactive mixtures produced even more violent results: Stoichiometric mixtures of $\mathrm{Cu}$ and $\mathrm{S}$ detonated at applied axial load of $2 \mathrm{GPa}$ (even without applied shear), producing $\mathrm{CuS}$. More exothermic reactions such as $\mathrm{Al} / \mathrm{Fe}_{2} \mathrm{O}_{3}$ proceeded in a detonationlike manner, damaging parts of the press - this reaction was initiated at hydrostatic pressure between 1-3 GPa, even without application of shear.

Russian scientists actively continued the work of Bridgman. Enikolopyan and co-workers studied many systems, both endothermic and exothermic in Bridgman anvils and high-pressure extruders [2-10]. They expanded the list of compounds that were originally investigated. Thermite mixtures of $\mathrm{Al}$ and $\mathrm{Fe}_{2} \mathrm{O}_{3}$, pressed into discs of thickness $4 \mathrm{~mm}$, reacted completely within $100 \mathrm{~ms}$ [7,9] (which, if nucleated from a side would correspond to a velocity as large as 40000 $\mathrm{m} / \mathrm{s}$ ). The anvil was destroyed and the lack of plastic deformation in its fracture zones points to a detonation. Particles were ejected from cylindrical preforms (samples were not radially contained) at velocities up to $2000 \mathrm{~m} / \mathrm{s}$ [11]. Reactions were accompanied by the emission of light and highenergy electrons, acoustic emission, and gamma radiation [5]. The experiments of Enikolopyan lend further proof to the existence of structural collapse. In order to explain the required level of mixing for these rates, the reactions must have been preceded by decomposition steps that consist of either a phase change (solid to liquid or supercritical fluid) or mechanical disintegration (pseudovolumetric fracture) or a combination of them. The results for the thermite reaction is particularly intriguing, since the particle sizes are so large 
(300 $\mu \mathrm{m} \leqslant \phi_{\mathrm{Al}} \leqslant 1000 \mu \mathrm{m} ; \phi_{\mathrm{Fe}_{2} \mathrm{O}_{3}} \approx 1000 \mu \mathrm{m}$ ) that the system would otherwise have difficulty to ignite and react in the normal self-heat-sustained mode. To explain such conversion rates on the basis of diffusion and thermal conduction only, the reacting particles would have to be clusters of 5-6 molecules.

Fast decomposition of a metastable phase under strain or through the coupling between chemistry and mechanical strain has also been observed in glassy semiconductors and metals, as well as in a geological context $[12,13]$. The "Prince Rupert drops," and more generally, tempered glasses under high-strain condition, which explode as a result of a mechanical stimulation $[13,14]$ is a spectacular example, which has remained a mystery for several centuries. Studies of detonation of classical solid explosives (such as heavy metal azides, which are compounds bearing the group $\mathrm{N}_{3}$ ) also reveal the existence of a fast propagation regime with velocities of the order of the sound velocity in solid matrices, before the gas explosion stage [15]. The explosive nature of recrystallization of amorphous materials has been described by Koverda [16]. Hlavacek [17] has observed a clearly distinguishable thermal wave when intensely milled aluminum powder transforms from amorphous (and highly plasticized) state to polycrystalline state. Fortov and co-workers [18] applied high current densities to thin $\mathrm{Nb}-\mathrm{Ti}$ wires in a cryostat. They have measured propagation velocities, for the transformation from the superconduction to the normal conduction phase of $10-12 \mathrm{~km} / \mathrm{s}$.

In a different context, it has been shown that chemical waves propagate at very low temperature $[19,20]$ and at usual temperatures [21] at rather high velocities, due to a coupling between chemistry and mechanical deformations. In the context of cryochemistry of solids, there is evidence of a transition between slow and fast heat-mechanochemical wave modes and possibly gasless detonation [22]. This physical phenomenon may be very important, as the fast autowave concept may help to explain the mystery of fast chemical evolution of substances in the universe [23]. It has also been proposed that catastrophic geotectonic phenomena, such as earthquakes, may be triggered by gasless detonation processes of phase transformations in the earth's crust (for example, explosive decay of a metastable glassy state of rocks to a more stable, polycrystalline phase) $[24,25]$. This hypothesis of phase transformations of rocks induced by a high value of the strain may resolve a number of difficulties with the current purely mechanical theory of earthquakes [24].

The experimental results described above strongly suggest the importance of a coupling between chemistry and mechanical deformations. Batsanov [26] pointed out that, with increased pressure, ionic compounds rearrange valence electron density distributions and the Szegeti charges (i.e., the actual degree of ionization of the atoms in ionic crystals) are reduced until a state of decomposition is reached. Gilman has shown that shear strain changes the symmetry of a molecule or of a solid and is thus effective in stimulating reactions, much more so than isotropic compression [27-29]. The reason is that a shear strain displaces electronic band energies in a different way, thus leading in general to a nar- rowing of the band gap separating the valence bands from the conduction bands. When the gap closes, the semiconductor solid becomes metallic that triggers a strong chemical reactivity. This process belongs to the class of phenomena grouped under the term "mechanochemistry" [30] and is also at work in the motion of a dislocation by that of kink (leading to plastic deformation). Indeed, the motion of a kink is akin to a local chemical reaction in which an embedded "molecule" is dissociated, and then one of the product atoms joins with an atom from another dissociation to form a new "molecule" [28]. Gilman has also proposed [31] that intense strain deformation (which, therefore, leads to bending of atomic bonds) occurs in a very narrow zone of atomic scale that can propagate at velocities comparable to or even higher than the velocity of sound in the initial material.

\section{PREVIOUS WORKS ON SHOCK WAVES AND EXPLOSIONS}

\section{A. The limits of conventional theory of shock waves and of explosions and the need for nonequilibrium mechanochemistry}

The initiation and propagation of shock waves have been studied for many years and the mainstay of theoretical description is still the macroscopic Rankine-Hugoniot equations, augmented by the Chapman-Jouguet $(\mathrm{CJ})$ processes [32]. This theory has proven to be remarkably accurate despite its simplicity, but it has certain restrictions and over the years the theory has evolved and it has seen some modifications. For example, the Chapman-Jouguet process yields a constant value for the velocity of the reaction front with respect to the unreacted phase. Sano and Miyamoto [33] have developed an unsteady state Rankine-Hugoniot theory and pointed out the deficiencies of the steady state theories. But arguably the greatest shortcoming of the theory to account for the above mentioned experiments is the lack of detailed description of events that occur on the microscopic level. Erpenbeck [34] combined molecular dynamics (MDS) and Monte Carlo methods to study diatomic exchange reactions and showed qualitative agreement with the CJ theory. An interesting finding of that study is the relatively long time required to reach equilibrium. This raises the question of nonequilibrium reactions, specifically in the setting of a detonation. White and co-workers [35] analyzed a similar system by MDS and their findings also confirmed the validity of the $\mathrm{CJ}$ theory only once equilibrium is established. Considerable effort has been focused on excitation and nonequilibrium in molecular crystals, due to their importance as secondary explosives. Dlott and Fayer [36] and Kim and Dlott [37] showed that, during the incipient phase after shock loading, bulk phonon modes are excited first and then energy is transferred to intramolecular modes through doorway modes that are most conducive to the transfer. In the case of naphtalene, equilibrium is only reached $200 \mathrm{ps}$ after a $40 \mathrm{kbar}$ impact. Coffey $[38,39]$ studied the interaction of molecules with compressive waves and developed a model for multiphonon excitation of intramolecular vibrational modes. These models have proven invaluable in the interpretation of experimental results under conditions where thermodynamics equilibrium 
is reached at the microscopic scale. However, as summarized in Sec. I, there are now an increasing number of experiments which cannot be understood within the conventional framework.

\section{B. The two fundamental mechanisms}

Two mechanisms have recently been identified as potential candidates for explaining very fast phase transformation and explosions in solids: (1) The products are mechanically stronger than the reactants leading to mechanical shock waves $[25,40,41]$; (2) the released energy is sufficiently large and its release rate is so fast that its transfer to microscopic degrees of freedom literally boosts and propels atoms to collide against each other leading to supersonic chemical fronts $[29,31,25]$.

Courant and Friedrichs [32] have studied wave propagation of finite amplitude in elastic-plastic materials and pointed out that shocks are not possible when the stressstrain characteristics of plastic material is of the weakening type. Sornette [25] adapted this one-dimensional (1D) formalism to study the opposite case in which the products are more elastically rigid than the reactants, and the density is smaller for the products than for the reactions, leading to a larger sound velocity for the products. Under these rather special conditions, a shock develops that propagates at a velocity intermediate between the acoustic wave velocities of the reactions and products. Consider a bar of material deformed uniformly with an initial strain everywhere along it. Suppose that a localized perturbation or inhomogeneity produces a local deformation larger than the initial strain at the left boundary of the bar. Qualitatively, the density perturbation will start to advance to the right in the product phase. Since the velocity is larger in the product phase, the largest deformations propagate the fastest. An initial smooth disturbance will progressively steepen and a shock will eventually form. The shock is fundamentally due to the stiffening transformation from reactants to products. The importance of this condition was independently recognized by Pumir and Barelko [40,41], using a slightly different formulation. Their framework coupling the elastic wave equation to a reactiondiffusion equation allowed them to reveal the existence of supersonic modes of deformations with the existence of a critical strain necessary to ignite gasless detonation by local perturbations.

The second mechanism discussed by Gilman [29,31] is illustrated in Fig. 1 (see also, Ref. [25]). A one-dimensional chain is made of atoms of mass $m$ linked to each other by energetic links of spring constant $k$, which when stressed beyond a limit, rupture by releasing a burst of energy $\Delta g$ converted into kinetic energy transmitted to the atoms. Initially, the chain of atoms is immobile. Suppose that the first atom on the left is suddenly brought to a position that entails the rupture of the first bond. This rupture releases the energy $\Delta g$, which is converted into kinetic energies of the atom fragment that is expelled to the left and of the next atom to the right that becomes the new left-boundary of the chain. Now, due to the impulsive boost $(\Delta g / m)^{1 / 2}$ that the boundary atom received, obtained by assuming that the released
Initial energetic crystalline structure
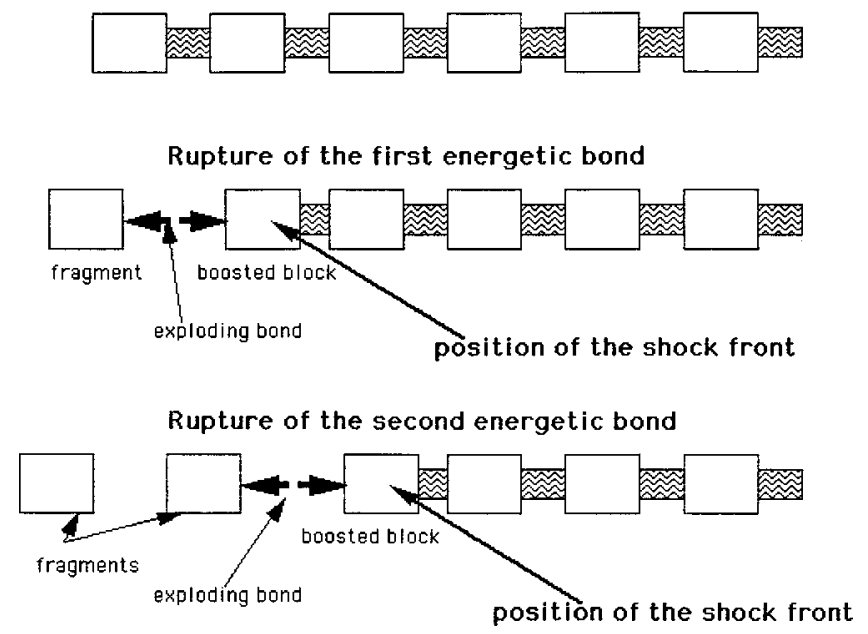

FIG. 1. A one-dimensional chain made of blocks linked to each other by energetic links, which when stressed beyond a given deformation threshold, rupture by releasing a burst of energy converted into kinetic energy transmitted to the blocks. The figure shows two successive bond ruptures that lead to velocity boosts to the ejected fragments on the left and to the boundary blocks.

energy $\Delta g$ is equally divided between the expelled fragment and the next atom to the right, this boost will eventually stress the bond linking it to the next atom towards its rupture threshold. When this occurs, it is expelled by the energy that is released and the next atom forming the new boundary is itself boosted suddenly by the amount $(\Delta g / m)^{1 / 2}$. It is then clear that this leads to a shock propagating at a velocity larger than the sound velocity equal to $c=(\mathrm{k} / \mathrm{m})^{1 / 2}$ in the long wavelength limit, since the atoms are receiving boosts that accelerate their motion faster than what would be the propagation by the springs with the usual acoustic wave velocity. Taking the continuous limit, Gilman [28] proposes that the resulting supersonic shock velocity $U$ is given by the Einstein formula $U^{2}=c^{2}+\Delta g$. This approximate semiempirical formula assumes that the bond energy would be entirely or almost entirely converted into the boosts with close to $100 \%$ efficiency, an assumption that can be tested by comparison of the predicted shock velocity with experiments [28]. Furthermore, it does not distinguish between particle and wave velocities and its theoretical status is unclear. It, however, appears to have empirical merits and we mention it here also because of its intuitive underpinnings. We also stress that the basic element of the model is not a unique atom and its bond to the next atom, but rather a supra-atomic element comprising at the minimum the unit cell linked to the next, in general, by several noncolinear bonds. This is similar to the mesoscopic modeling strategy developed below. Therefore, the compression of the effective bond from one cell to the next corresponds to additional shear and rotational deformation modes of the cells.

The validity of the second mechanism has also been explored with a one-dimensional microscopic model for the propagation of a detonation specially adapted to solids [42]. It couples a nonlinear equation for the elastodynamics of the 
crystal lattice to an equation describing the molecular exothermal chemical reaction. An essential ingredient of the model is that the chemical reaction sustains the shock by giving a positive acceleration to the atoms, consistent with the second mechanism discussed previously [25,29,31]. In return, the shock is assumed to promote the chemical reaction by the effect of the violent mechanical distortions that are responsible for strong forces acting on the molecules. When the strength of the positive feedback of the shock on the chemical reaction is beyond a threshold, the detonation is found to switch from a low-energy low-speed regime to a high-energy high-speed regime. The earlier model for the propagation of a detonation in a molecular solid $[43,44]$ also captured the property of pumping kinetic energy into the system by bond rupture. This model with a two-dimensional lattice of diatomic molecules connected by Morse or Lennard-Jones potential with a predissociative exothermic intramolecular potential gives detonations that propagate as coherent waves that are resistant to thermal fluctuations. Extensions to three-dimensional molecular dynamics and to inhomogeneous energetic crystals are found respectively in Refs. [45] and [46].

\section{MODELING STRATEGY USING THE REACTIVE TODA LATTICE}

The understanding of the conditions under which these two mechanisms will be active in real materials is at a rudimentary stage, not to speak of their possible interplay. We need models that allow us to explore the relationship between nonequilibrium processes at the microscopic scale, the chemical reactions, and the possible development of supersonic shocks and explosions. To achieve this goal, we propose to use a mesoscopic approach describing how the microscopic processes self-organize into dynamic macroscopic structures of patterns and waves. The fundamental problem, and therefore, the challenge, in any mesoscopic model lies in the compromise between scale (macroscopic limit) and detail (microscopic limit). The compromise proposed here is based on the Toda lattice [47], which is a one-dimensional system of entities interacting through an interaction potential limited to nearest neighbors. Using a coarse-grained discrete lattice allows us to perform simulations on large systems at times large as compared to all the relevant times scales so that the characteristics of the self-organization behavior can be studied in details.

The advantage of the Toda lattice are multifold. First, for small deformations corresponding to small deviations of the position of the atoms from the bottom of the potential wells, linear elasticity is recovered. In this regime, the acoustic waves (phonons) are nondispersive in the large wavelength and small amplitude limits. Its nonlinear potential at large deformation leads to mode coupling, which, as we shall briefly discuss, gives naturally the Maxwell-Boltzmann distribution of energies. Thus, with a purely deterministic dynamics (Newton's equations) on a minimal model, we have a basic thermodynamics that we can then enrich to study chemical reactions coupled with mechanical strain. The Toda lattice has dispersion at nonvanishing frequencies, which is adequate to capture the effect of the microscopic atomic structure as well as any possible mesoscopic organization. The Toda lattice has been extremely well studied in the literature as a remarkably simple system exhibiting stable localized collective excitations, called solitons. The Toda model is known as the only integrable one in atomic lattices but we do not use this remarkable property.

Solitons are a particular set of a more general class of solitary waves or moving discrete breathers found in many lattice systems with both dispersion and anharmonic interactions [48]: the dispersion tends to disperse the modes while the nonlinearity tends to concentrate them. The resulting behavior is a localized coherent nonlinear wave. We stress that our choice of the Toda potential does not restrict our conclusions as similar solitary waves can be observed for a large class on nonlinear potentials. In particular, we have checked that Taylor expansions of the exponential terms in the Toda potential truncated at different orders do not change our results at the qualitative level. We think that the results reported below are robust to a generalization of the Toda potential. Indeed, recently, the existence of solitons has been established in anharmonic lattices for a large class of interatomic potentials $[49,50]$. It is true that our use of a onedimensional model favors the existence of solitary waves. However, nonlinear classical Hamiltonian lattices are well known to exhibit generic solutions in the form of discrete breathers [48], which are time periodic and typically exponentially localized in space. Necessary conditions for their occurrence are the existence of upper bounds on the phonon spectrum of small fluctuations around the ground state of the system as well as the nonlinearity. Nonstable but propagative long-lived local concentration of energy are also often found and should play a similar qualitative role as the one played by solitons in the present model.

We modify the classic Toda model to include metastable states and the possibility for a phase transformation or chemical reaction. Specifically, beyond a certain strain threshold, the potential felt by the particles of the lattice is modified to represent a change of phase from the reactants (initial lattice) to the products. In contrast to regular dispersive waves, solitons act as energy traps because they create a dynamic state where the local energy flux points in the direction of wave propagation. As a consequence, the potential energy, which is released during the reaction as kinetic energy, can be trapped within the soliton, enhancing its localization and its velocity. This process occurs out-ofequilibrium, i.e., without equilibration with other degrees of freedom and can thus focus energy to extremely high levels. In our knowledge, a Toda lattice with chemical reaction has not been studied before.

More generally, the Toda lattice should also shed light on several issues in ultrafast solid phase reactions that are currently not understood. When the shock wave travels through a particle, either fracture or melting must occur, because the high reaction rates can only be explained by the generation of large surface areas. Toda [47] mentions that the Toda lattice has a property called "chopping phenomenon." When a soliton, which is a compressive pulse, is reflected at a free boundary a temporary tensile pulse develops that could be 
causal in spalling or fracture near the free boundary. Since the tensile soliton is unstable, it degenerates into a series of pulses and there is a rapid transfer of energy from the soliton to thermal vibrations of the lattice.

\section{THE CLASSIC TODA LATTICE (TL) MODEL}

Here, we introduce the classic Toda lattice (without reaction) and investigate the lattice equilibrium. Local departures from equilibrium are demonstrated for solitons in inert lattices (with no metastable states) and the problem of nonequilibrium is discussed.

\section{A. Definition}

The Toda lattice is a model of a one-dimensional chain of atoms. Consider a one-dimensional lattice consisting of $N$ particles. Each particle is described by a point of mass $m_{n}$ that only interacts with neighboring masses. Hence, heterogeneities, discontinuities, pores, and perfectly isotropic states are all defined by the pairwise values of the interaction potential and masses. The displacement of the $n$th mass from its equilibrium position is $y_{n}(t)$. The relative displacement is defined as $r_{n}=y_{n+1}-y_{n}$. The lattice motion is described by the following canonical equations:

$$
m \frac{d^{2} r_{n}}{d t^{2}}=\phi^{\prime}\left(r_{n+1}\right)-2 \phi^{\prime}\left(r_{n}\right)+\phi^{\prime}\left(r_{n-1}\right),
$$

where $\phi^{\prime}$ denotes the first derivative of the potential function with respect to the relative displacement. In his search for an integrable lattice that also exhibits realistic mechanical behavior, Toda used a recursive formula to find periodic and single soliton solutions. As a result, the potential function

$$
\phi(r)=\frac{b}{a} e^{-a r}+b r \quad(a b>0),
$$

was proposed. Application of this choice in Eq. (1) gives the Toda lattice (TL). Note that the integrability of the Toda lattice makes this system (1) special because it exhibits by construction an infinite number of invariants $[47,48]$. The TL equations can also be written in terms of the displacement,

$$
m \frac{d^{2} y_{n}}{d t^{2}}=b\left[e^{-a\left(y_{n}-y_{n-1}\right)}-e^{-a\left(y_{n+1}-y_{n}\right)}\right] .
$$

While the TL model is 1D and mesoscopic in nature, we still want to use parameters that are realistic. As an illustration, we use the properties of $\mathrm{Al}$ to determine the parameters in Eq. (3). If $a=1 / \lambda$, where $\lambda$ is the athermal lattice constant $=4.5 \AA$ and the cold longitudinal sound velocity is $c_{0}$ $=6420 \mathrm{~m} / \mathrm{s}$, it follows that $b=4.1 \times 10^{-9} \mathrm{~N}$. The model is written in nondimensional form: $t \rightarrow t c_{0} / \lambda$ and $y \rightarrow y / \lambda$. Later, we will look at the effect of chemistry in a simplistic manner by changing the parameters of the potential function (more details in Sec. V). Let $a^{\prime}$ and $b^{\prime}$ be the new parameter values due to the chemical reactions that will be introduced in Sec. V. We define the dimensionless parameters $\alpha$ $=a^{\prime} / a, \beta=b^{\prime} / b$. Thus, $\alpha=\beta=1$ are the values used to model the standard (nonreactive) lattice. The nondimensional TL equation becomes

$$
\frac{d^{2} y_{n}}{d t^{2}}=\beta\left[e^{-\alpha\left(y_{n}-y_{n-1}\right)}-e^{-\alpha\left(y_{n+1}-y_{n}\right)}\right] .
$$

\section{B. Energy partitioning in the Toda lattice}

\section{The Maxwell-Boltzmann distribution}

If the TL is a fair description of a chain of atoms and nonlinear springs, one would expect that in the absence of external forcing, the lattice should approach a thermodynamic equilibrium state. We specify below what we mean with respect to the known organization of TL and for the application to the chemical reactions discussed below.

A first approach would consist of defining the modes of vibrations and investigating the partition of total energy between them. This approach is prone to difficulties since all atomic degrees of freedom are coupled anharmonically by the Toda potential. Thus, the definition of an elementary wave oscillator as an extended quasiparticle is somewhat arbitrary. Actually as a consequence of integrability, the Toda Hamiltonian decouples exactly in terms of two kinds of traveling excitations, namely, cnoidal waves that are the anharmonic counterpart of phonons and aperiodic solitons. As these quasiparticles have infinite lifetimes (in infinite systems), they cannot decay into one another so that thermalization is ultimately precluded in the strict sense by integrability. Any configuration is bound to recur in future time at least quasiperiodically and is thus not consistent with the Maxwell-Boltzmann distribution.

However, having in mind using the TL as a mesoscopic representation of a system undergoing a fast chemical reaction, we would like to define a local effective temperature describing the agitation of local masses in their local nonlinear potential. If we can show that there is the analog of an approximate Maxwell-Boltzmann distribution for the local degrees of freedom of the vibrations, we will be able to contrast this "thermal" agitation with the more coherent and much faster motions involved in the explosive chemical reaction. We will be able to conclude that the chemical reaction is mostly athermal, i.e., mostly controlled by solitary structures. In such a fast out-of-equilibrium chemical reaction, notwithstanding the observation of energy equipartition of the local vibrations over long time scales, the chemical reactions will be seen to occur so fast that only solitary structures can contribute significantly to them.

To achieve this goal, we term "oscillator" a mass with its kinetic energy plus local nonlinear potential energy. The TL is thus a chain of "oscillators." The question we ask is whether a local subset of oscillators that are initially more excited should exchange energy with neighbors so that in the end the Maxwell-Boltzmann distribution is recovered for the distribution of energy carried by these local oscillators. It is well known that the TL model is not ergodic (due to the integrability property), but that does not exclude energy sharing. We show below that this state is described by the Maxwell-Boltzmann distribution for the translational degrees 


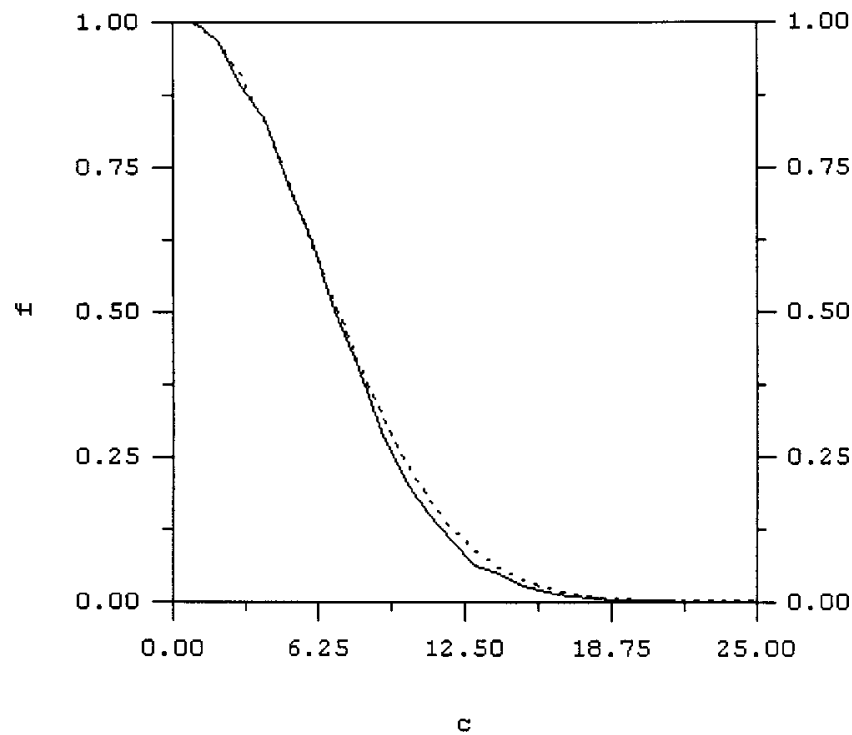

FIG. 2. Maxwell-Boltzmann distribution (--) and TL distribution of energies carried by local "oscillators" defined as single mass plus their local nonlinear potential energy. The results have been obtained for a chain of 201 oscillators integrated over a total reduced time equal to 500. We use the nondimensional TL equation (4) (with $\alpha=\beta=1$, since chemistry is not considered here). Therefore, the energies are dimensionless.

of freedom. Indeed, since the total energy of the system is conserved (in the absence of dissipation), this corresponds to the microcanonical ensemble and the relevant observables are the energy of the microscopic degrees of freedom.

We denote the instantaneous velocity of an oscillator as $c$ and decompose it into a drift or wave component $w$ and a random fluctuating component $\nu$. When the oscillations are resolved on the time scale associated with the Debye frequency (which is basically the period of a thermal vibration), the question is how do we discriminate between motion associated with thermal fluctuation and motion associated with an event like a wave that propagates through the lattice? The oscillator does not discriminate, it only responds to the immediate force, be it of thermal or mechanical (wave) origin. The numerical results of Fig. 2 pertain to a situation where no external force was applied, in other words, we did not have a drift component $w$ and the velocity of each oscillator was taken to be $v$. The stochasticity was introduced through the initial conditions of the oscillators; the initial conditions were zero velocity for all oscillators and random displacement between $[-W,+W]$, where $W$ is an input parameter for the system. The larger $W$, the larger the input energy. The Toda lattice was then integrated for these random initial displacements and zero velocities.

If the chain is in equilibrium, it has a Maxwell-Boltzmann distribution parameterized by $T$. This means that the probability to find a state of energy $E$ is $e^{-E / k T}$, where $k$ is the Boltzmann constant (whose sole use is to convert a temperature scale into an energy scale) and $T$ is the (temperature) parameter that quantifies the degree of excitation or disorder of the vibrations within the lattice. Using the form (2) for the potential energy, the Maxwell-Boltzmann distribution reads

$$
f=A e^{-2 \gamma\left[\Phi+(1 / 2) v^{2}\right] / T} \quad \text { with } \quad \gamma=\frac{m c_{0}^{2}}{2 k}(\text { units K), }
$$

where we have used the expression of the total energy of a given oscillator (a spring + mass) as the sum of the potential energy stored in the spring and the kinetic energy associated with the stochastic component of the mass velocity.

The dimensionless potential function is defined as

$$
\Phi=\frac{\beta}{\alpha}\left[e^{-\alpha\left(y_{n+1}-y_{n}\right)}-1\right]+\beta\left[y_{n+1}-y_{n}\right] .
$$

This distribution (5) should be calculated by counting the number of oscillators in a given energy bin. For this, we integrate the nondimensional TL equation (4) (with $\alpha=\beta$ $=1$, since chemistry is not considered here) and construct the histogram of the energy of individual oscillators using the cumulative statistics over all the elements in the lattice and over time. Assigning random initial displacements of the lattice points uniformly distributed in $[-W, W]$, the density distribution of the oscillators over energy space has the form shown in Fig. 2 where it is compared with the MaxwellBoltzmann distribution. $W$ is thus the characteristic scale of the energy put initially inside the TL. Figure 2 has been constructed with the choice $W=0.081$ that is best matched by the Maxwell-Boltzmann distribution at the temperature $T=297 \mathrm{~K}$. By performing several runs, we verify that $T$ is proportional to $W$, indicating that higher input energy results in higher temperature. This is an important test of our model. In addition, we kept the boundaries free in these numerical experiments and observed thermal expansion. It is actually quite interesting that the linear expansion that occurs compares quite favorably with the linear (volumetric/3) expansion coefficient of aluminum (recall that the $a$ and $b$ values of the potential correspond to aluminum).

To further test for the relevance of the MaxwellBoltzmann distribution, we have also constructed the distribution over a single oscillator (for instance the 50th oscillator) and by summing the statistics over a time interval $\Delta t$. As $\Delta t$ increases, we verify that the energy distribution of a single oscillator is also well-described by the MaxwellBoltzmann distribution. This result implies that the stochastic component of a typical oscillator velocity added to its potential exhibit an approximate ergodicity property.

These results recover those of Saito et al. [51] who found that, for finite chains bounded by two reflecting walls with strong nonlinearity, an ergodic state occurs corresponding to strong stochasticity, which is consistent with the prediction of Israilev and Chirikov [52].

\section{Nonequilibrium configurations}

The quest for equipartition of energy and for the Maxwell-Boltzmann statistics from nonlinear dynamics was first initiated by Fermi, Pasta, and Ulam, who failed [53]. More recent works (see, for instance, Ref. [54]) have shown the subtlety of this problem. In their pioneering work, Fermi, Pasta, and Ulam revealed that even in strongly nonlinear one-dimensional classical lattices, recurrences of the initial 
state prevented the equipartition of energy and consequent thermalization. The questions following from this study involve the interrelations between equipartition of energy (Is there equipartition? In which modes?), local thermal equilibrium (Does the system reach a well-defined temperature locally? If so, what is it?), and transport of energy/heat (Does the system obey Fourier's heat law? If not, what is the nature of the abnormal transport?). The surprising result of Fermi, Pasta, and Ulam has now been understood: under general conditions for classical many-body lattice Hamiltonians in one dimension, it has been shown that total momentum conservation implies anomalous transport in the sense of the divergence of the Kubo expression for the coefficient of thermal conductivity [55]. The anomalous transport is thus a specific feature of one-dimensional systems. Thus, our verification of an approximate Maxwell-Boltzmann distribution does not prevent the existence of anomalous transport or propagation properties as we discuss in the sequel.

It is indeed possible to perturb a small region of the lattice in such a way that these nodes depart from the equilibrium distribution and this perturbation propagates with conservation of form and of energy. If the chain contains many nodes, the nonequilibrium state of the small number of nodes perturbed in this coherent mode (namely, forming a soliton) will not significantly alter the overall distribution. The transit time of a soliton over a given oscillator is very small compared to the time scale over which equilibrium is achieved at the scale of a single oscillator. The transit of a soliton is thus fundamentally a nonequilibrium process. To recognize this fact is essential for our investigation of the coupling with a chemical reaction.

Indeed, when a shock wave propagates through the lattice, the distribution of the energies of the oscillators in the shock zone is perturbed away from its Boltzmann distribution. Depending on the magnitude of the shock (ranging from a sound wave to a detonation), the degree of deviation from equilibrium could vary between insignificant to complete. Quantifying this degree of deviation from equilibrium constitutes one of the major dilemmas of shock theory: within the shock zone, nonequilibrium could exist, temperature could become meaningless and a macroscopic description of chemistry with Arrhenius kinetics becomes nonsensical. Our mesoscopic approach allows us to investigate precisely this regime and the interplay between the equilibrated degrees of freedom and the out-of-equilibrium impulses.

To study the effect of an external perturbation, we consider a force that is applied at the first node,

$$
F_{L}=M \sin \left(\frac{\pi t}{t_{I}}\right), \quad t \leqslant t_{I} \quad \text { and } \quad F_{L}=0 \quad \text { for } t \geqslant t_{I}
$$

in a system of $n=200$ masses and springs. At the other end of the chain, a mass is held immobile, corresponding to fixed boundary conditions. We identified the presence of a soliton by observing a pulse of amplitude above the noisy background that propagates at a velocity slightly above $1 \mathrm{M}$. The same procedure will be used below in Sec. III for the other cases.

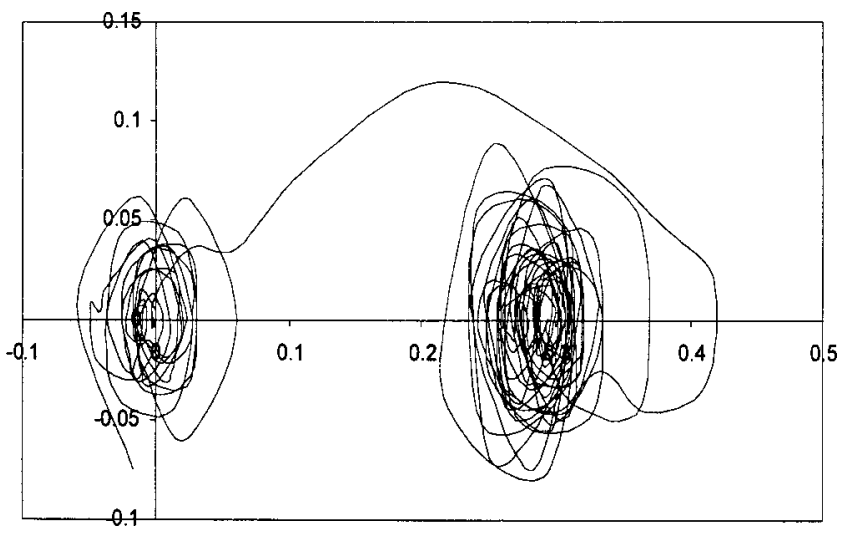

FIG. 3. Phase diagram for the 70th oscillator showing dimensionless velocity obtained by integration of the nondimensional TL equation (4) as a function of displacement from the equilibrium position before (left chaotic region) and after (right chaotic region) a strong impact of the oscillator by a solitary wave. The abscissa is the position measured from the initial equilibrium and the ordinate is the velocity of the particle.

The impulse (7) corresponds to a single arch starting from and returning to 0 . The motion of the first node is then described by

$$
\frac{d^{2} y_{0}}{d t^{2}}=\beta\left[1-e^{-\alpha\left(y_{1}-y_{0}\right)}\right]+F_{L} .
$$

The last node could be either fixed or unbounded. When the force is small, the deviation from equilibrium is slight. Application of a stronger force at the first node will cause the creation of a solitary wave leading to a strong deviation from equilibrium as illustrated in a phase diagram for the 70th oscillator shown in Fig. 3. The conditions and parameter values for this example are listed in Table I as case 1. The oscillator has been in equilibrium before the first arrival of the shock wave as can be seen from the chaotic trajectory in

TABLE I. $M$ is the maximum amplitude of the force impulse applied at one extremity of the chain. $t_{I}$ is the duration of the forcing applied at the boundary that creates a train of solitary waves disturbing the equilibrium. The parameter $\lambda\left(\lambda_{f}\right)$ is the lattice constant of the reactants (the products if there is a transformation). When both are equal (i.e., $\lambda / \lambda_{1}=1$ ), there is no chemical reaction and the simulations correspond to the classic Toda lattice. The parameters $\alpha_{f}$ and $\beta_{f}$ are the ratio of the two parameters defining the Toda potential taken for the reactions over those taken for the products. The values $\alpha_{f}=\beta_{f}=1$ correspond to the absence of any chemistry, i.e., to the standard Toda lattice. $r_{c}$ is the threshold for compression at which the phase transformation or chemical reaction is triggered.

\begin{tabular}{lcccccc}
\hline \hline Case no. & $M$ & $t_{I}$ & $\lambda_{f} / \lambda$ & $r_{c}$ & $\alpha_{f}$ & $\beta_{f}$ \\
\hline $\begin{array}{l}\text { 1: No } \\
\text { reaction }\end{array}$ & 0.5 & 1 & 1 & & 1 & 1 \\
$\begin{array}{l}\text { 2: No } \\
\text { reaction }\end{array}$ & 0.5 & 10 & 1 & & 1 & 1 \\
3 & 0.5 & 10 & 0.9 & -0.3 & 1.5 & 3.0 \\
4 & 0.5 & 10 & 1.1 & -0.2 & 0.9 & 0.8 \\
5 & 0.5 & 10 & 1.1 & -0.2 & 1.1 & 1.5 \\
\hline \hline
\end{tabular}




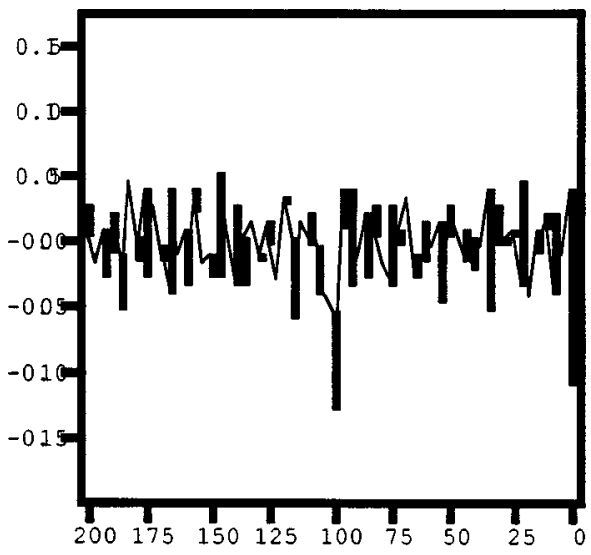

(a)

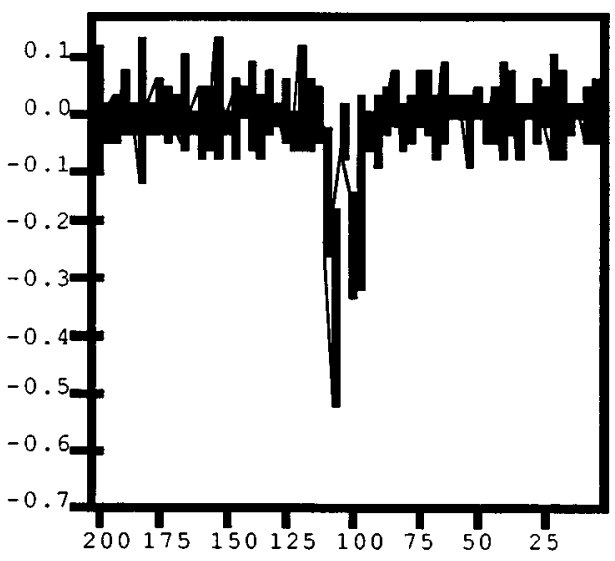

(b)
FIG. 4. Strain profiles at time $t=100$ of case 1 (a) and case 2 (b) showing, respectively, 1 and 2 solitons propagating over a noisy "thermally" equilibrated background. The position along the $x$ axis is given in units of the mesh size. phase space shown in Fig. 3. When this oscillator is shaken by the passage of the shock, it rapidly moves away from its equilibrium state to a novel state, a new position in phase space where it settled chaotically and equilibrates over time. Due to the finiteness of the system used in the simulations, the shock will reach the boundary of the lattice and be reflected at the last node (we use a fixed boundary condition at the extremity $n=200$ and a free boundary at the other extremity). When the 70th oscillator is again shaken by the reflected solitary wave, it undergoes a second translation. Between these strong perturbations, the oscillators can reach equilibrium provided the time intervals between the solitary wave perturbations are much longer than a vibrational period. In this example, the 70th oscillator started with a temperature of $297 \mathrm{~K}$ before the solitary wave arrived the first time and finished with the temperature of $328 \mathrm{~K}$ after the solitary wave was completely dissipated and final equilibrium was restored globally in the lattice. Before this final equilibrium was reached, the oscillator had been displaced several times by the weakening multiply reflected solitary wave. These results are consistent with previous numerical analysis by Hill and Knopoff [56], who showed that strong shocks in strongly nonlinear TL are led by a soliton, are unstable and have a relatively chaotic state immediately behind the shock front. They also found that the frequency and velocity distributions of infinite lattices are singular and inconsistent with the vibrational properties of a thermodynamically equilibrated crystalline solid at nonzero temperature.

In the next example (case 2), the duration $t_{f}$ of the applied force is extended ten times such that it becomes slightly larger than twice the dimensionless natural period $2 \pi / \sqrt{2 \alpha \beta}$ of oscillation of the oscillators, obtained by expanding Eq. (4) to linear order and thus neglecting dispersion. The strain (relative displacement) profiles along the lattice of cases 1 and 2 are shown in Fig. 4 at $t=100$. There is a distinct difference between the two profiles. In case 1, the solitary wave travels as a single perturbation through the medium with velocity close to $1 \mathrm{M}$. In case 2 , the initial perturbation has split into two solitary waves. The leading one has a velocity of $1.08 \mathrm{M}$ and the second wave travels at $1.02 \mathrm{M}$. As a consequence, as time progresses, the distance between the two waves of case 2 increases. The solitary waves in case 2 conserve their form as they pass through each other, consis- tent with the original studies of Zabusky and Kruskal [57], defining them as "solitons." At the free boundary, the solitons are destabilized and their energy is transferred to the lattice. The energy near the free boundary thus increases after the soliton has been reflected and a "hot spot" is generated.

The system was integrated until equilibrium at which the temperature finally reached by the system was $517 \mathrm{~K}$ in case 2 compared to $328 \mathrm{~K}$ in case 1 . The higher temperature of case 2 is expected, since considerable more energy has been injected into the system. An interesting observation is the creation of multiple waves whose number is given by the integer of $t_{f} / 5$. For $t_{f}$ less than 5 , no solitary structure was observed, only strong concentrated acoustic waves propagating at $1 \mathrm{M}$ and dissipating within the lattice at they propagate. When the (dimensionless) impact time are 5, 10, and 20, one, two, and four solitons are formed, respectively. Another interesting result is the effect of impact strength $M$ defined in Eq. (7). When $M$ decreases to zero (regardless of impact time), the perturbation does not split into separate solitons. Instead, it travels as a single perturbation whose velocity approaches $1 \mathrm{M}$. This derives from the well-known fact that the propagation velocity of a Toda soliton decreases as the amplitude of its associated strain field decreases and eventually equates the sound velocity for vanishing amplitude. The relationship between amplitude and velocity is completely determined by the parameters defining the Toda model.

When the impact strength is small, the system is adequately described by a linear system (linearization of the exponential terms) and the velocity of any wave is determined by material properties alone. When the impact strength increases, the nonlinear interaction becomes prominent and the wave speed is no longer determined by material properties alone, but also by the magnitude of the impact. This reflects the fundamental property of solitons and more generally of solitary waves to result from the competition between dispersion (which tends to have waves at different frequencies propagate at different velocities) and nonlinearity (which tends to concentrate waves into sharp shocks).

The stability of solitons and the instability of dark solitons are thoroughly investigated in the literature (cf. [47]). Solitons keep energy focused in a small region of the chain and 
the energy does not disperse to the rest of the chain- $\mathrm{a}$ curiously stable, albeit nonequilibrium state. This is a clear indication that energy transfer in the soliton must occur exclusively in the direction of propagation. The energy flux is only directed in the direction of propagation. This property is essential for understanding our results reported below of the coupling between the mechanical deformation associated with solitary waves and chemical reactions occurring in the lattice. Indeed, if chemical energy is released in the soliton and if it retains its coherent nondispersive localized structure, it is possible to focus energy within this solitary wave to very high levels.

\section{CHEMICAL REACTION IN THE TODA LATTICE}

We now define and study the reactive lattice undergoing a chemical transformation. This model can be easily generalized to an axisymmetrical 2D geometry. The terms "chemical transformation" and "chemical energy" are used in a loose sense. We envision the following experimental situation in which a mechanical system can suddenly undergo a phase transformation or a chemical reaction when its local mechanical deformation reaches a threshold. This can occur, for instance, according to the mechanisms of Batsanov [26] and Gilman [27-29] in which the distortion of the lattice by shear strain moves the electronic bands, leading eventually to a closing of the band gap (metallization) and, therefore, to a sudden strong chemical reactivity. In our system, we shall account for the existence of a phase transformation or of a chemical reaction by introducing a potential energy source when the relative displacement (strain) $r_{n}=y_{n+1}-y_{n}$ at some point in the lattice reaches a critical threshold $r_{c}$. Thus, when two nodes are sufficiently compressed so that the relative displacement $r_{n}$ becomes smaller than the threshold $r_{c}$, we assume that the initial system (reactants) is transformed into a new phase (products) characterized by different parameters for the potential function defined in the right-hand side of Eq. (4), as shown in Table I. Note that the transformation occurs and the "chemical energy" is released only when the material is in the compressed state. In this description, the released chemical energy could refer to the energy difference between phases, to an amorphous-crystalline transformation, or a chemical transformation. Chemical reaction between different species necessarily involves the issue of mixing, which is not addressed in the present study. In our discrete model, the reaction nucleus is the size of the lattice mesh. Recall that a mass and its local environment are not describing the atomic scale but an effective mesoscopic scale. This implies that the physical size of the nucleus is no larger than the mesh size. A genuine description of the critical nucleus would require a more complex three-dimensional Hamiltonian with a microscopic spatial resolution.

The general result established in statistical physics, that there are no equilibrium phase transitions in one dimension, is not relevant to this work. This is because we define a chemical phase transition from a phenomenological point of view involving an effective description of the many degrees of freedom embodied by a single element at the mesoscopic level. In other words, our model is not one-dimensional from a microscopic point of view. Recall that a similar remark has already been formulated above with respect to the difference between the nature of the strain at the mesoscopic level and that at the atomic level.

Some complication may occur as a chemical reaction brings additional time scales into the system. Here, we assume that the time scale for the release of chemical energy is much shorter than the time scales involved in the wave propagation. To investigate the interplay between mechanical waves and chemical energy release at a phenomenological level, the interaction potential is irreversibly changed into the following expression:

$$
\begin{aligned}
\Phi_{f}= & \frac{\beta_{f}}{\alpha_{f}}\left\{\exp \left[-\alpha_{f}\left(y_{n+1}-y_{n}+\frac{\lambda-\lambda_{f}}{\lambda}\right)\right]-1\right\} \\
& +\beta_{f}\left[y_{n+1}-y_{n}\right],
\end{aligned}
$$

when $r_{n}$ becomes smaller than $r_{c}$. The lattice constant $\lambda_{f}$ of the product state could be smaller or larger than $\lambda$. Three different cases are investigated, the first case (case 3 in Table I) describes a product that is denser (i.e., $\lambda_{f}<\lambda$ ), the second case (case 4 in Table I) describes a material with larger lattice constant $\left(\lambda_{f}>\lambda\right)$ and smaller sound velocity, and the third case (case 5 in Table I) describes a product with larger lattice constant and larger sound velocity than the initial material. The parameters are listed in Table I. (The interaction potentials before and after the reaction are made continuous at $r=r_{c}$ by adding a constant to $\Phi_{f}$.)

Case 3 (cf. Table I): $\lambda_{f}=0.9 \lambda$. We introduce an initial perturbation that is strong enough to initiate the chemical reaction. The time evolution of the reaction is presented in Fig. 5 in a set of six sets of two panels: The panels on the left show the displacements as a function of distance along the 1D-lattice for six different times increasing from top to bottom. The panels on the right show the corresponding chemical conversion profile ( 0 corresponds to the initial chemical composition; 1 means full conversion to the products of the chemical reaction). Acoustic waves created by the initial perturbation and the triggered chemical reaction propagate supersonically through the medium in an apparently random way, similar to a thermal wave. The leading part of this wave is always compressive. However, this compressive state weakens along the propagation and about midway through the lattice, the conversion criterion is not met. The conversion halts and an ordinary acoustic wave continues to propagate through the remainder of the lattice with no further conversion. However, there is a subtle component of the vibration that is going to play a key role in the future evolution of the system.

The two top panels on the left of Fig. 5 correspond to time $t=58$, at which a small soliton is ready to be spawned from the leading edge of the chemical reaction wave. At this same moment $(t=58)$, the conversion profile exhibits a sharp front located at $x=95$ (traveling towards the left boundary at $x=200$ ).

At time $t=70$, the relative displacement along the lattice allows us to distinguish clearly the existence of the soliton that has been spawned by the reaction front. At about this 

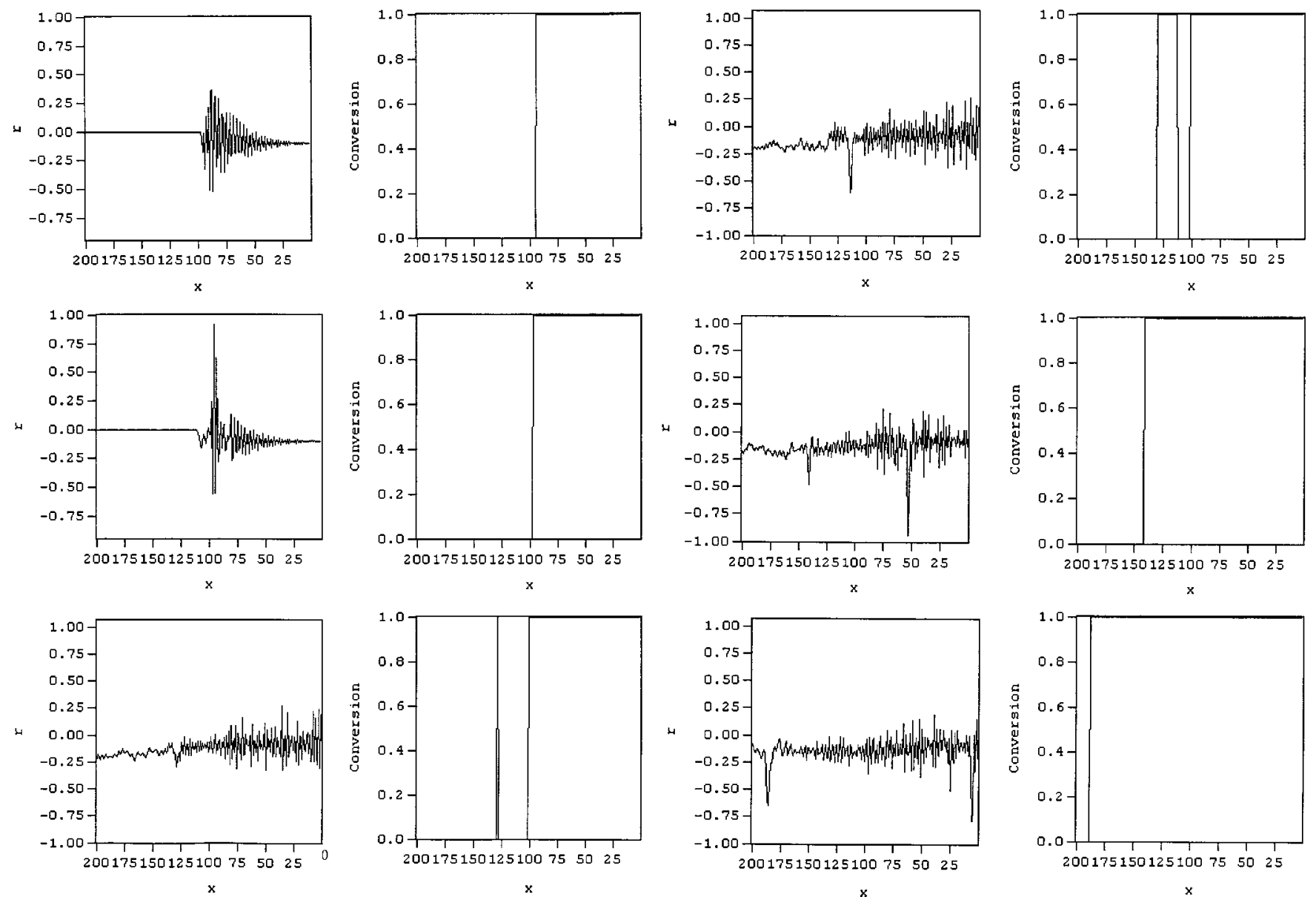
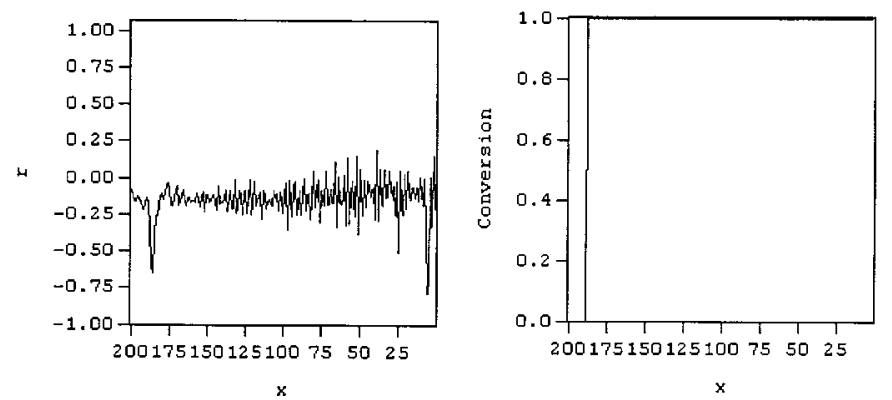

FIG. 5. Relative displacement and conversion profiles. Top to bottom and left to right, $t=58,70,229,235,256$, and 276. The position along the $x$ axis is given in units of the mesh size.

time, the reaction front comes to a standstill at $x=102$. The stagnation of this front is due to the fact that the compression-criterion for triggering the chemical reaction is no longer met at the boundary between reacted and unreacted material.

However, the soliton continues its journey all the way to the left border at $x=200$, where it is reflected from the fixed boundary to travel back towards the static chemical reaction front. Small acoustic waves spin off from the stalled front and propagate towards $X=200$ and the approaching soliton.

The soliton runs through this noisy displacement field and at $t=229$ the soliton is at $x=129$. The conversion criterion is met at this point and chemistry is turned on. The position $x$ $=129$ corresponds to a point where the soliton superimposed with a local compressive region of the "acoustic wave" gives a stress just large enough to initiate chemistry.

The panels at $t=235$ show the soliton traveling towards the stalled reaction front at $t=229$. The new reaction front triggered by this soliton is now propagating with it to the right towards the other stalled front. At time $t=240$, the new front runs into the stalled front and no unreacted material is left between the fronts.

We now have basically the same situation as at time $t$ $=58$, when the front was located at $X=95$. The system now spawns another little soliton at $x=129$ at $t=252$. That means that between $t=240$, when the two fronts consolidated, and $t=252$, no conversion happened and no front movement was discernible. But the system was breeding another little soliton during this pause period.

In the next panels at $t=258$, the new little soliton that has spun off from the reaction front at $x=129$ is shown to be at $x=141$. It travels again towards the left boundary at $x$ $=200$. The chemical reaction front is now traveling together with the soliton. The conversion profile at $t=258$ is also located at $x=141$.

Complete conversion is established after $t=282$ when the soliton and the reaction front simultaneously collide with the left boundary of the lattice.

In summary, we have shown that the chemical reaction can be fully completed after an initial triggering perturbation, only because of the presence of solitons launched from the reaction front that interact with the ambient energetic acoustic field radiated by the reaction front. We have also shown that a soliton can support a reaction front. The simulations have demonstrated the existence of a remarkable interaction between the soliton, the ambient acoustic field, and the reaction front. Note that the scenario shown in Fig. 5 is only one among many similar histories that depend on the initial conditions and the level of preexisting ambient acoustic noise.

As we have seen, the reaction front propagation can be explained in terms of the solitons existing for unreacted and 


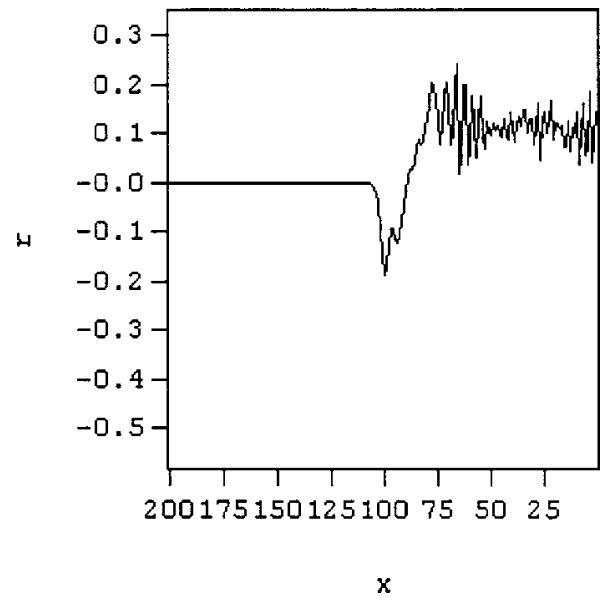

FIG. 6. Strain profiles for case 4. The position along the $x$ axis is given in units of the mesh size.

product solids without reaction. With $\left(\alpha_{f}, \beta_{f}\right)=(1.5 ; 3.0)$ fixed for the unreacted solid (then $r_{c}$ becomes irrelevant) and setting $\lambda_{f}=\lambda$, we indeed found solitons for perturbations $M$ and $t_{I}$ values as listed in Table I. For $\left(\alpha_{f}, \beta_{f}\right)=(0.9 ; 0.8)$ and $\left(\alpha_{f}, \beta_{f}\right)=(1.1 ; 1.5)$, we also found solitons. These solitons differed in the time to develop and their relative positions with respect to each other. The stochastic wave pattern over which the solitons superimposed can probably be described in terms of cnoidal waves corresponding to extended strain field. Note that both the cnoidal and soliton excitations are supersonic and their propagation velocity increases with the strain amplitude.

Case 4 (cf. Table I): $\lambda_{f}=1.1 \lambda$ with a smaller sound velocity. A soliton is not observed for this case. The strain profile in Fig. 6 shows an expansion of the lattice behind the wave front. Therefore, the conversion is interrupted soon after onset because the compression cannot be sustained to a level that is strong enough. It is clear that the energy that is released in the reaction does not contribute towards sustaining and strengthening of the compression in the leading part of the wave. Beyond the point where the criterion for the chemical transformation is no longer met, the wave propagates as an acoustic wave through the system and the conversion profile becomes frozen.

Case 5 (cf. Table I): $\lambda_{f}=1.1 \lambda$ with a larger sound velocity. A solitary wave is formed in the reactive lattice and it

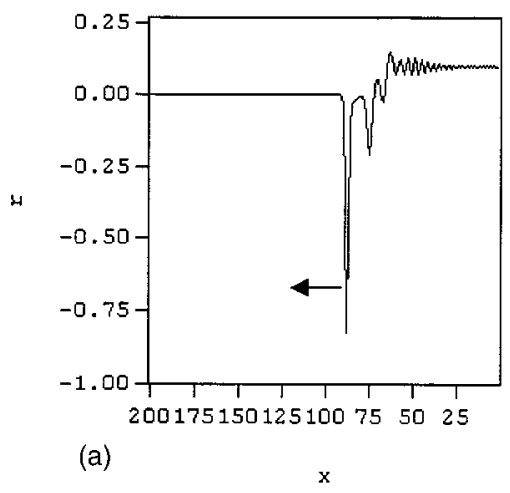

appears to have all the properties of a soliton. Of course, it is not a genuine soliton since its front separates two different phases, with the products left behind it and the reactants in front of it. All the chemical potential energy that is released during the reaction remains trapped in the solitary wave, which travels supersonically with respect to the sound velocity in the reactant solid. Both the compression and particle velocities in the wave increase, thus the released chemical energy is stored as kinetic and elastic potential energy. This case has the same qualitative properties as expansion shocks in conventional fluid mechanics, where the conditions needed to obtain an expansion shock are the simultaneous increase in velocity and in volume. These conditions constitute quite unusual circumstances that are generally viewed as curiosities (Fig. 7).

Note that, after its reflection from the fixed boundary at $n=200$, the solitary wave has "consumed" all the reactions and the entire system consists of the new phase or products. In this new phase, it becomes a bona fide soliton, traveling unaffected through the other waves in the product material. No further chemical transformations occur and the soliton now travels with constant speed.

\section{DISCUSSION}

We have presented a numerical study that suggests that solitary waves may play an important role in explosive supersonic reactions and conversions. For this, we started from the classic TL, extending it beyond its use as a phenomenological model for processes occurring at the atomic level. Since most of experimental observations are performed at the mesoscopic and macroscopic levels, we use the TL as a phenomenological model that embodies the minimal number of essential ingredients of the problem (nonlinear elasticity+dispersion) to explore the different possible regimes. We stress that the phenomenology documented and summarized in this paper for the TL is qualitatively in agreement with that obtained from a continuous description based on the Boussinesq equation that can be further transformed into the Korteweg de Vries equation. We have then extended the TL to include chemistry in order to describe a reactive material in nonequilibrium situation, with applications to explosions and detonations as well as very fast phase conversion.

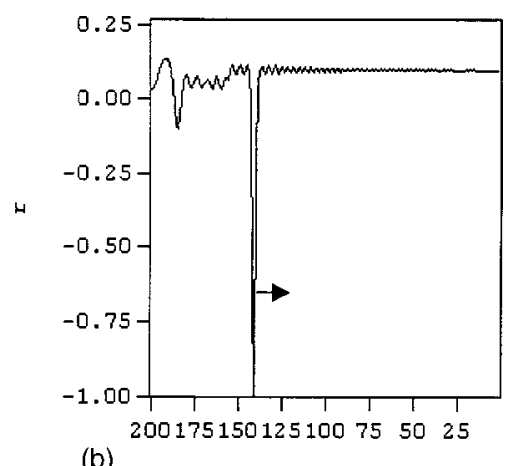

(b)

FIG. 7. Strain profiles for case 5 at (a) $t=60$, (b) $t=160$. The position along the $x$ axis is given in units of the mesh size. 
The TL equations are deterministic but the oscillators clearly adopt the Boltzmann distribution, thus providing the correct analog of a thermodynamics. The equilibrium state has been calculated by the probability of finding a given velocity state space both using an ensemble statistics over a large number of oscillators at a fixed moment in time and by cumulative statistics over a long period of time for a single oscillator (effective ergodicity property). We have clearly documented that the oscillators approach the Boltzmann distribution at long times, that the state variables are defined and they can be matched with the continuum model.

When perturbations as in the shock wave occur on time scales too short to allow the oscillators to reach the Boltzmann distribution, one needs a model that takes suitably into account both equilibrium and out-of-equilibrium states and their interactions. In this sense, the TL is ideally suited. We have, in addition, built-in chemistry by modifying the Hamiltonian at reactant interfaces to a double well potential: When a compressive stress threshold is reached, reactants combine irreversibly to give products that are modeled by a different interaction potential. Chemical energy is released when the mechanical vibrations resulting from both the possible existence of a solitary wave and of the rest of the vibrational background forces a node to reach the conversion threshold and thus crosses the energy barrier.

We have observed that the released chemical energy initially contributes to the kinetic energy of the oscillator. Due to friction (as in the Langevin equation), this energy tends to be transformed over time into vibrational energy or heat in the transition zone behind the shock wave. If solitary waves can be nucleated by the initial conditions, we have shown that they act as traps of energy: In the presence of ongoing chemical energy release, the energy piles up within the trapping solitary wave, providing a positive feedback that enhances the strength and velocity of the solitary wave. The trapping property of solitons and of solitary waves result from the fact that the energy flux within them points forwards in the wake. In contrast, the energy flux points backwards in the wake of ordinary dispersive waves resulting in a negative feedback. Thus, dispersive waves in nonreactive media dampen out, but in the presence of chemistry, the chemical energy released in the wave competes with energy losses in the wake to result in either a steady reactive wave or an attenuating wave that extinguishes. We believe and propose that the relevant point is not so much the existence of solitons in the strict sense, but the possibility of transient but long-lived coherent propagative concentration of energy which can contain and concentrate the energy released by the chemical concentration for a sufficiently long time. Of course, we have not proved that this is the case in dimen- sions larger than one, but offer it as a conjecture to be tested in future works.

When chemistry is included in the lattice, a nonsteady state solitary wave is observed for two cases: (1) when the product expands with respect to the reactant and the system has higher sound velocity and (2) when the product is more compact that the reaction. The first case involves a rather peculiar combination of conditions that is a curiosity rather than a genuinely realistic situation. The second case is much more interesting and we have shown a complex interplay between the solitons, acoustic waves, and reaction fronts. The situation thus seems much richer and more complex than obtained previously in Ref. [25] and in Refs. [40], [41]. As long as reactants are available, it converts the material and traps the chemical energy as elastic potential energy and the balance as kinetic energy. Within the spatial zone in which the chemical reaction actually takes place, the lattice is highly compressed and particles have large particle velocities. These velocities belong to the drift component and not the random (or thermal) component of the total velocity. The chemical transformation is athermal as the system is far from equilibrium. Furthermore, the chemical energy is not directly released as thermal energy. No energy spills over into thermal energy as long as free boundaries or internal defects are absent. At defects and free boundaries, conversion to thermal energy occurs and eventually leads to thermal equilibrium at long times.

When the product has a larger lattice constant and lower sound velocity, a supersonic wave is observed directly after impact. But it is not self-sustaining and disappears, leaving only an acoustic wave. There results seem to hold true in all the simulations we have performed until now which explored a significant part but nevertheless nonexhaustive fraction of the phase diagram.

The simple model proposed here allows us to study nonequilibrium chemistry and lattice motions with great ease. The limitations of this model must also be recognized, especially its one dimensionality and the interactions that are limited to nearest neighbors. Although 3D MDS are computationally costly, they should be used to verify the findings presented here. This study is underway. Finally, it would be extremely interesting to develop experimental techniques to probe directly for the existence of the long-lived propagative concentrations of energy that we propose here.

\section{ACKNOWLEDGMENTS}

This work was partially supported by the NSF through Grant No. CTS-0096381 (H.J.V) and by NSF-DMR9971475 and the James S. McDonnell Foundation (D.S.).
[1] P. Bridgman, J. Phys. Rev. 48, 825 (1935).

[2] N. S. Enikolopyan, Dokl. Akad. Nauk SSSR 283, 612 (1985).

[3] N. S. Enikolopyan, A. A. Mkhitaryan, and A. S. Karagezyan, Dokl. Akad. Nauk SSSR 288, 436 (1985).

[4] N. S. Enikolopyan, Russ. J. Phys. Chem. 63, 1261 (1989).
[5] N. S. Enikolopyan, A. A. Mhkitaryan, A. S. Karagezyan, and A. A. Khzardzhyan, Dokl. Akad. Nauk SSSR 292, 121 (1987).

[6] N. S. Enikolopyan, V. B. Vol'eva, A. A. Khzardzhyan, and V. V. Ershov, Dokl. Akad. Nauk SSSR 292, 177 (1987).

[7] N. S. Enikolopyan, A. A. Khzardzhyan, E. E. Gasparyan, and 
V. B. Vol'eva, Dokl. Akad. Nauk SSSR 294, 567 (1987).

[8] N. S. Enikolopyan and A. A. Mkhitaryan, Dokl. Akad. Nauk SSSR 309, 900 (1989).

[9] N. S. Enikolopyan, Dokl. Akad. Nauk SSSR 302, 862 (1988).

[10] N. S. Enikolopyan, A. I. Aleksandrov, E. E. Gasparyan, V. I. Shelobkov, and A. A. Mkhitaryan, Dokl. Akad. Nauk SSSR 319, 612 (1991).

[11] J. S. Olcott, Science 140, 1189 (1963).

[12] V. A. Shklovskii, Zh. Eksp. Teor. Fiz. 82, 536 (1982).

[13] C. Meade and R. Jeanloz, Nature (London) 339, 616 (1989).

[14] T. V. Polyakova, I. M. Fomin, and D. N. Karpinskii, Akad. Nauk 311, 431 (1993).

[15] V. V. Barelko, S. M. Ryabykh, K. Sh. Karabukaev, Sov. J. Chem. Phys. 12, 377 (1994).

[16] V. P. Koverda, Tech. Phys. 39, 255 (1994).

[17] P. Pranda, K. Prandova, and V. Hlavacek, Combust. Sci. Technol. 156, 81 (2000).

[18] V. E. Fortov, V. L. Gershenkroi, V. L. Dorofeev, and Yu. D. Kuroedov, Dokl. Phys. 43, 484 (1998).

[19] V. V. Barelko, I. M. Barkalov, V. I. Goldanskii, D. P. Kiryukhin, A. M. Zanin, Adv. Chem. Phys. 74, 339 (1988).

[20] V. V. Barelko, I. M. Barkalov, V. I. Goldanskii, A. M. Zanin, and D. P. Kiryukhin, Russ. Chem. Rev. 59, 205 (1990).

[21] N. S. Enikolopyan, Dokl. Akad. Nauk SSSR 283, 897 (1985).

[22] D. P. Kiryukhin, P. S. Mozhaev, and V. V. Barelko, Khim. Fiz. 11, 264 (1992).

[23] V. V. Barelko, I. M. Barkalov, V. I. Goldanskii, D. P. Kiryukhin, and A. M. Zanin, Adv. Chem. Phys. 74, 339 (1988).

[24] D. Sornette, Phys. Rep. 313, 238 (1999).

[25] D. Sornette, in Earthquake Thermodynamics and Phase Transformations in the Earth's Interior, edited by R. Teisseyre and E. Majewsi (Cambridge University Press, Cambridge, England, 2000), Vol. 76, pp. 329-366.

[26] S. S. Batsanov, Russ. J. Inorg. Chem. 36, 1265 (1991).

[27] J. J. Gilman, J. Mater. Res. 7, 535 (1992).

[28] J. J. Gilman, Philos. Mag. B 67, 207 (1993).

[29] J. J. Gilman, Czech. J. Phys. 45, 913 (1995).

[30] J. J. Gilman, Science 274, 65 (1996).

[31] J. J. Gilman, Philos. Mag. B 71, 1057 (1995).

[32] R. Courant and K. O. Friedrichs, Supersonic Flow and Shock
Waves (Interscience, New York, 1948).

[33] Y. Sano and I. Miyamoto, J. Appl. Phys. 84, 6606 (1998).

[34] J. J. Erpenbeck, Phys. Rev. A 46, 6406 (1992).

[35] J. W. Mintmire, D. H. Robertson, and C. T. White, Phys. Rev. B 49, 14859 (1998).

[36] D. D. Dlott and M. D. Fayer, J. Chem. Phys. 92, 3798 (1990).

[37] H. Kim and D. D. Dlott, J. Chem. Phys. 93, 1695 (1990).

[38] C. S. Coffey, Phys. Rev. B 32, 5335 (1985).

[39] C. S. Coffey and E. T. Toton, J. Chem. Phys. 76, 949 (1982).

[40] A. Pumir and V. V. Barelko, Eur. Phys. J. 10, 379 (1999).

[41] A. Pumir and V. V. Barelko, Eur. Phys. J. 16, 137 (2000).

[42] P. Maffre and M. Peyrard, J. Phys.: Condens. Matter 6, 4869 (1994).

[43] M. Peyrard, S. Odiot, E. Lavenir, and J. M. Schnur, J. Appl. Phys. 57, 2626 (1985).

[44] M. Peyrard, S. Odiot, E. Oran, J. Boris, and J. M. Schnur, Phys. Rev. B 33, 2350 (1986).

[45] S. G. Lambrakos, M. Peyrard, E. S. Oran, and J. P. Boris, Phys. Rev. B 39, 993 (1989).

[46] P. Maffre and M. Peyrard, Phys. Rev. B 45, 9551 (1993).

[47] M. Toda, Theory of Nonlinear Lattices, Springer Series in Solid-State Sciences Vol. 20 (Springer, Berlin, 1981)

[48] S. Flach and C. R. Willis, Phys. Rep. 295, 182 (1998).

[49] G. Friesecke and J. A. D. Wattis, Commun. Math. Phys. 161, 391 (1994).

[50] J. Szeftel, P. Laurent-Gengoux, E. Ilisca, and M. Hebbache, Physica A 288, 225 (2000).

[51] N. Saito, N. Ooyama, Y. Aizawa, and H. Hirooka, Prog. Theor. Phys. 45, 209 (1970).

[52] F. M. Israilev and B. V. Chirikov, Sov. Phys. Dokl. 11, 30 (1966).

[53] E. Fermi, I. R. Pasta, and S. M. Ulam, Los Alamos National Laboratory Report No. LA-1940, 1955 (unpublished).

[54] R. Livi, M. Pettini, S. Ruffo, M. Sparpaglione, and A. Vulpiani, Phys. Rev. A 31, 1039 (1985).

[55] T. Prosen and D. K. Campbell, Phys. Rev. Lett. 84, 2857 (2000).

[56] T. G. Hill and L. Knopoff, J. Geophys. Res. 85, 7025 (1980).

[57] N. Zabusky and M. D. Kruskal, Phys. Rev. Lett. 15, 240 (1965). 


\title{
Modeling of a-Si : $\mathrm{H}$ deposition in a de glow discharge reactor
}

Dariusz Orlicki and Vladimir Hlavacek

Laboratory for Ceramic and Reaction Engineering, Department of Chemical Engineering, State

University of New York at Buffalo, Buffalo, New York 14260

\author{
Hendrik J. Viljoen ${ }^{\text {a) }}$ \\ Departmen of Chemical Engineering, University of Nebraska-Lincoln, Lincoln, Nebraska 68588-0126
}

(Received 16 July 1991; accepted 17 April 1992)

\begin{abstract}
PECVD reactors are increasingly used for the manufacturing of electronic components. This paper presents a reactor model for the deposition of amorphous hydrogenated silicon in a de glow discharge of $\mathrm{Ar}-\mathrm{SiH}_{4}$. The parallel-plate configuration is used in this study. Electron and positive jon densities have been calculated in a self-consistent way. A macroscopic description that is based on the Boltzmann equation with forwardscattering is used to calculate the ionization rate. The dissociation rate constant of $\mathrm{SiH}_{4}$ requires knowledge about the electron energy distribution function. Maxwell and Druyvesteyn distributions are compared and the numerical results show that the deposition rate is lower for the Druyvesteyn distribution. The plasma chemistry model includes silane, silyl, silylene, disilane, hydrogen, and atomic hydrogen. The sensitivity of the deposition rate toward the branching ratios $\mathrm{SiH}_{3}$ and $\mathrm{SiH}_{2}$ as well as $\mathrm{H}_{2}$ and $\mathrm{H}$ during silyl dissociation is examined. Further parameters that are considered in the sensitivity analysis include anode/cathode temperatures, pressure, applied voltage, gap distance, gap length, molar fraction of $\mathrm{SiH}_{4}$, and flow speed. This work offers insight into the effects of all design and control variables.
\end{abstract}

\section{INTRODUCTION}

Plasma enhanced CVD (PECVD) has gained prominence as an important material processing lool since its introduction in the mid $60^{\prime} \mathrm{s}$. In the PECVD process, gases are ionized by an electric energy source to form a plasma. Through the intermediation of the highly energized electrons, radical species are formed from the precursor molecular gases. These radicals are chemically very active, and their stability in the gas phase is determined by the rate of recombination and disproportionation reactions. The more stable radicals diffuse to the reactor walls where they are depleted by rapid insertion reactions. One outstanding feature of the PECVD process is the ability to energize electrons to very high levels, without any significant rise in the gas temperature. Processing at lower substrate temperature holds several advantages; e.g., temperature-sensitive material can be used, and a reduction in residual stress results, due to thermal misrnatch.

Whereas traditional CVD systems employed thermal energy to assist the deposition reaction, PECVD uses electrons produced by the glow discharge. The advantages of PECVD are obvious, but the understanding of and the ability to control this system are considerably more complex than thermal CVD processes. Historically, PECVD was developed for microelectronic applications.

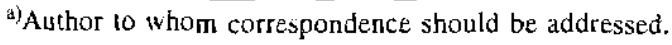

Passivation layers, diffusion masks, and interlayer dielectrics can be deposited at low temperatures. But the major advantage of PECVD wals realized when macroelectronic devices were labricated by this method. Examples are photovoltaic cells, large area display panels, linear arrays, and other thin film based technology.

Recently PECVD was used to deposit thin films of polyerystalline diamond.' Microwave plasmas are normally used for diamond deposition, but they have certain limitations. If a PECVD process can be devised that produces a large concentration of atomic hydrogen and sufficient ion bombardment to remove any pyrolytic carbon, it has a good chance to deposit diamond-like film at a fast rate. This brings us to the philosophy of a generic reactor design. Different objectives will lead to differem designs. For example, if it is desirable to have a large concentration of atomic hydrogen, gap space and electric field strength are the crucial factors; if a uniform deposition rate is the objective, then the hydrodynamic design will require more altention and in industrial reactors, conversion and yield are the decisive factors in the design process.

The glow discharge decomposition of silane to produce amorphous hydrogenated silicon $(a-S i: H)$ is the most well-known PECVD process. It is important because of its use in solar cells and thin film transistors. ${ }^{2}$ The Japanese have also embarked on a program to scale-up plasma processes to achieve time and cost reduction in device fabrication through mass production. 
In particular, their 'Sunshine Project' ${ }^{\cdot 3}$ is aimed at the development of low-cost mass production techniques for fabricating a-Si:H solar cells. Another crucial development was the discovery in 1975 that the electronic properties of the glow discharge deposited material could be controlled very effectively by substitutional doping from the gas phase. ${ }^{4}$ This possibility has opened a rapidly growing new field of research and applications.

The motivation for this work is to develop an integrated model that can be used to predict film growth rate and uniformity and analyze the sensitivity of these qualities. The model finds important applications in the design, scale-up, and characterization of PECVD reactors. The model consists of two parts; the first part models the glow discharge and the second part describes the plasma chemistry and the spatial distribution of the neutral species.

A macroscopic approach was followed to derive the model for the glow discharge. This implies that electron and ion behavior are described by a one-dimensional continuum model and the rate of ionization is determined by a nonequilibrium, macroscopic relation with the electric field. The electron and ion density distributions, the electric field, and the rate of ionization then give a selfconsistent description of the glow discharge and these equations are solved numerically. Space discretization was done according to the Scharfetter-Gummel scheme. Starting from an estimated solution, it was improved by implicit integration of a false transient form of the equations. The steady state solution was then calculated by Newton's method.

For the second part of the model, a two-dimensional compressible fluid flow model was used to calculate the concentration of all neutral species, and the ideal gas law was used as the equation of state. A Galerkin finite element method was used to calculate the velocity, temperature, and concentration fields, and the deposition rates and profiles along the electrode surfaces were also calculated. The model also made provision for the temperature and pressure dependencies of all the physical properties. Special consideration was given to the physical properties of the atomic hydrogen and the radicals. But the kcystone of the process is, of course, the electron-silane dissociation reactions, and little will be gained by paying attention to the other details while neglecting this step.

The dissociation of silane produces silyl and silylene. But the formation of silylene also occurs along two possible routes; in one case hydrogen is produced and in the other case molecular hydrogen is produced. Distinction between these two routes is important since atomic hydrogen participates in a secondary hydrogen extraction reaction with silane to form silyl. The experimental collision cross section for dissociation does not distinguish among the different dissociation reactions, and branching among the three possible reactions introduces two additional parameters to the system. The rate of dissociation warrants some discussion, since it plays such an important role. The main disadvantage of any macroscopic approach is the lack of information about the electron energy distribution (EEDF). The EEDF is required to calculate the dissociation rate. In this study we will assume two forms of the EEDF. namely the Maxwellian and the Druyvesteyn distributions. The mechanism of film growth is not perfectly clear, but it is known (cf. Kushner ${ }^{5}$ ) that silylene can insert directly into saturated silicon bonds on the surface, while silyl can insert only into a dangling bond (i.e., $\equiv \mathrm{Si}-\cdot$ ). The emission of hydrogen from the film underlies the insertion rates, but in this model sticking coefficients of unity were used for both sily! and silylene.

Deposition rates and profiles on both electrodes are calculated and compared for the different energy distributions. Furthermore, a parameter study was done where the values of all the parameters of the model were varied by $\pm 10 \%$ from a base case and changes in deposition rates and film uniformity were used to rank the sensitivity of all the parameters.

\section{THE MODEL}

A model that describes the behavior of a PECVD reactor consists of the conservation equations for charge, mass, energy, and momentum as well as the transport of charged and chemically active spccies. In this model a feeding gas of $\mathrm{Ar}$ and $\mathrm{SiH}_{4}$, where the silane concentration is in the order of $1 \%$, is considered. The addition of a molecular gas to the noble gas will affect the electron energy distribution. But we considered only the ionization of $\mathrm{Ar}$ in the model; since silane is not included in the glow discharge model, the chemistry/hydrodynamic part of the model becomes decoupled from the discharge with significant savings in computing times. To make this assumption more justifiable, we considered only mole fractions of silane in the range of $1 \%$.

\section{A. Glow discharge}

A continuum description for the behavior of the glow discharge is followed. The continuum description is valid only as long as certain criteria are met. The most important one is that the number of charged particles contained in a sphere of Debye radius is large.

$$
\begin{aligned}
n \times \frac{4}{3} \pi l_{D}^{3} & =n_{D} \gg 1 \\
l_{D} & =\left(\frac{\epsilon_{0} \mathrm{k}_{\mathrm{B}} T}{\rho_{c} e}\right)^{1 / 2}
\end{aligned}
$$

where $\epsilon_{0}$ is the permittivity of free space, $k_{B}$ is the Boltzmann constant, $\rho_{c}$ is the charge density, and $e$ 
is the charge of a particle. This condition limits the plasma charge granularity and ensures weak potential interactions.

Since $A r$ is an electropositive gas, the model will not include any anion formation; i.e., any inelastic collision between an electron and an Ar-atom will have a negligible probability to be of the associative type. The governing equations for the glow discharge are as follows.

\section{Electron conservation equation}

$$
\frac{\partial n_{e}}{\partial t}+\nabla \cdot j_{e}=\alpha j_{e} \frac{N}{N_{0}}
$$

This balance for the electron density $n_{e}$ considers only electron-neutral inpact ionization as a source term; due to the low degree of ionization the change in Arconcentration is very small and $N / N_{0}$ remains close to unity. Hence the glow discharge is decoupled from any neutral species balance.

\section{Positive ion conservation equation}

$$
\frac{\partial n_{p}}{\partial t}+\nabla \cdot j_{p}=\alpha j_{e}
$$

The fluxes $j_{e}$ and $j_{p}$ are defined for the potential $V$ as

$$
\begin{aligned}
& j_{e}=-D_{e} \nabla n_{e}+\mu_{e} n_{e} \nabla V \\
& j_{p}=-D_{p} \nabla n_{p}-\mu_{p} n_{p} \nabla V
\end{aligned}
$$

First it should be noted that the term for convective transport tacitly implies a momentum balance for the charged species of the form

$$
e_{k} E+\nu_{k m} m U_{k}=0, \quad k=e, p
$$

where $U_{k}$ is the velocity and in $e_{k}$ the sign of the charge should be taken into consideration. The mobility $\mu_{k}$ can be easily inferred from Eq. (7). $\nu_{k m}$ is a collision frequency for momentum transfer. Experimental values for mobilities and diffusion coefficients will be used in this model (see Appendix). The potential in the gap is given by the Poisson equation.

\section{Poisson equation}

$$
\nabla^{2} V=-\frac{|e|}{\epsilon_{0}}\left(n_{p}-n_{e}\right)
$$

The ionization coefficient $\alpha$ determines the rate of electron generation, and either a microscopic or a macroscopic approach can be followed. If the microscopic approach is followed, all swarm parameters, including $\alpha$, are determined by solving the Boltzmann equation. A less arduous approach is to determine $\alpha$ by a macroscopic approach. One possibility is to ascribe to the electrons a certain energy distribution (EEDF), e.g., a Maxwellian distribution, then the concept of electron temperature can be introduced and ionization is treated as in chemical reaction theory. Graves and Jensen ${ }^{6}$ used this method and assumed a kinetic form of the Arrheniustype. Segur et al. ${ }^{7}$ defined a memory kernel for their nonequilibrium model and fitted it to values for a $\mathrm{He}$ plasma. Friedland ${ }^{8,9}$ derived a nonequilibrium model, based on the forwardscattering form of the Boltzmann equation; i.e., the distribution function depends only on one spatial dimension and energy. Taking the first and second moments of the Boltzmann equation (with respect to energy) and using the assumptions that the ionization and excitation cross sections depend linearly on energy $\left(Q_{i}=a \epsilon, Q_{e}=k \epsilon\right)$, the following equation is found for $\alpha$ :

$$
\frac{d \alpha}{d z}+\alpha^{2}+p k \xi \alpha-p a E=0
$$

where $k$ and $a$ are the coefficients of the excitation and ionization cross sections, respectively, and $p$ is the total pressure. $\xi$ is the amount of energy that is lost by an electron in a collision and it is taken as the mean of the excitation and ionization values:

$$
\xi=\frac{1}{2}\left(\xi_{\text {ion }}+\xi_{\text {exs }}\right)
$$

The energy loss for excitation collisions $\left(\xi_{\text {exc }}\right)$ also presents an average of the different excitation collisions that are possible." The ionization rate is highest in the cathodic sheath region, and in this region the assumptions of the Friedland formulation hold best. Although the assumptions are no longer valid in the bulk plasma, the ionization is low and the error will not affect the model qualitatively. To improve the model will require a self-consistent microscopic description, but at the expense of excessive computing times.

There exists quite a variely of reactor configurations; the most popular are the parallel-plate type and the concentric cylinders with axial flow. The configuration that will be used in our model is a parallel-plate reactor, shown schematically in Fig. I. Inlet and outlet sections are provided for flow stabilization. Bised on this configuration, we will also assume that the charge species will have only unidirectional motion, and any axial advection will be negligible compared to the electric-field directed motion. Hence the Laplace and del-operators can be written as second and first derivatives with respect to $z$.

The boundary conditions used in the calculations are as follows:

The cathode $(-H / 2)$ is not transparent for the flux of positive ions and the flux of electrons is due to secondary emission.

$$
\left.\begin{array}{rl}
j_{e} & =-\gamma j_{p} \\
\frac{d n_{p}}{d z} & =0 \\
V & =-V_{D C}
\end{array}\right\} z=-\frac{H}{2}
$$




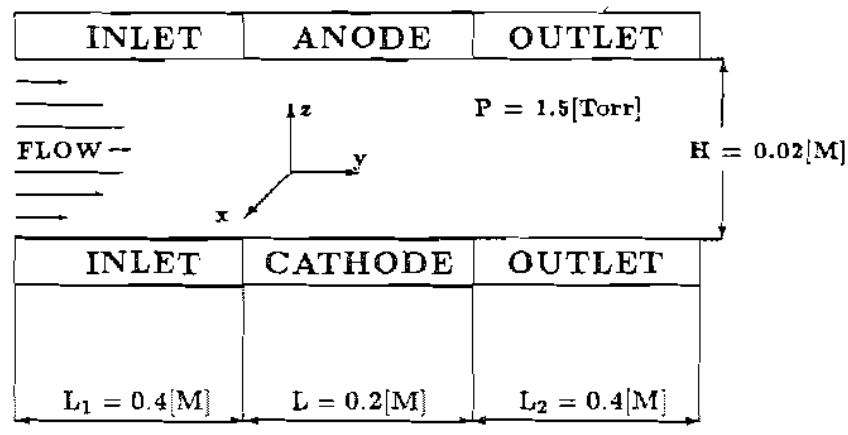

FIG. 1. Parallcl-plate rcactor schcma.

where $\gamma$ is the secondary emission coefficient.

The anode $(H / 2)$ perfectly absorbs electrons and the flux of positive charged particles is set to zero.

$$
\left.\begin{array}{rl}
\frac{d n_{p}}{d z} & =0 \\
n_{e} & =0 \\
V & =0
\end{array}\right\} z=\frac{H}{2}
$$

\section{B. Hydrodynamic model of reactor}

Gas flows through the reactor and the transport of neutral species are not affected by the electric field. The ionization in typical glow discharges accounts for a conversion of only one part per million and therefore the neutral species mass balances and momentum balances are decoupled from the rest of the plasma model. In order to describe the distribution and fluxes of neutral species, specifically the silane and radicals, a two-dimensional compressible flow model is used.

\section{Continuity equation}

$$
\nabla \cdot(\rho \mathbf{U})=0
$$

\section{Momentum equations}

$$
\rho \frac{D \mathbf{U}}{D t}=-\nabla \mathbf{p}-\nabla \cdot \bar{\tau}+p \mathbf{g}
$$

where $D / D t$ denotes the substantial derivative and $\nabla$ represents the divergence operator. The viscous tensor $\bar{\tau}$ is defined for a Newtonian fluid as

$$
\bar{\tau}=-\mu\left(\nabla \mathbf{U}+(\nabla \mathbf{U})^{\mathbf{T}}\right)+\frac{2}{3} \mu \nabla \cdot \mathbf{U I}
$$

In the energy conservation equation, the effects due to changes in pressure, viscous dissipation, and concentration gradients (Dufour effect) were neglected.

\section{Energy equation}

$$
\rho C_{p} \frac{D T}{D t}=\nabla \cdot \mathbf{k} \nabla T
$$

The system is closed by an equation of state, and for our operating conditions the ideal gas law was used.

\section{Equation of state}

$$
R_{g} T \rho=p \sum_{i=1}^{n} M_{i} x_{i}
$$

If the average molecular mass of the gas is assumed to be constant, the conservation equation for any species can be written as

\section{Conservation of mass}

$$
\begin{aligned}
C \frac{D x_{i}}{D t}= & \nabla \cdot\left[C D_{i}\left(\nabla \mathbf{x}_{\mathbf{i}}+\alpha_{\mathrm{i}} \mathbf{x}_{\mathrm{i}} \nabla \ln \mathbf{T}\right)\right] \\
& +\mathbf{R}\left(\sum_{\mathbf{j}} \mathbf{x}_{\mathrm{j}}\right)
\end{aligned}
$$

The choice of boundary conditions is also a point of contention, and these conditions must be chosen in such a way that they correctly describe the prevalent physical conditions at any boundary. The configuration that is used has fore- and after-section with the electrodes in between. The flow is then developed when it enters the fore-section and Poiseuille flow also prevails at the end of the after-section;

$$
\mathrm{U}=\left(0, U_{p}, 0\right)
$$

where $U_{p}$ denotes the Poiseuille solution. At solid walls the non-slip condition was applied

$$
\begin{aligned}
& \overline{\mathbf{n}} \cdot \mathbf{U}=0 \\
& \overline{\mathbf{t}} \cdot \mathbf{U}=0
\end{aligned}
$$

where $\overline{\mathbf{n}}$ and $\overline{\mathbf{t}}$ denote unit normal and tangential vectors to the solid walls. For the temperature and mass balance equations, the Danckwerts boundary conditions are used. At the inlet of the reactor

$$
\overline{\mathbf{n}} \cdot \mathbf{U} \rho C_{p}\left(T-T_{0}\right)=\mathbf{k} \overline{\mathbf{n}} \cdot \nabla \mathbf{T}
$$

$$
\overline{\mathbf{n}} \cdot \mathbf{U}\left(x_{i}-x_{i, 0}\right)=D_{i}\left(\overline{\mathbf{n}} \cdot \nabla x_{i}+\alpha_{i} x_{i} \overline{\mathbf{n}} \cdot \nabla \ln T\right)
$$

and at the outlet of the reactor section

$$
\begin{gathered}
\overline{\mathbf{n}} \cdot \nabla T=0 \\
\overline{\mathbf{n}} \cdot \nabla x_{i}+\alpha_{i} x_{i} \overline{\mathbf{n}} \cdot \nabla \ln T=0
\end{gathered}
$$

The surface on the upper part of the reactor system, which includes the fore-section, anode, and after-section, is kept at a constant temperature

$$
T\left(z=\frac{H}{2}\right)=T_{a n}
$$

but the temperature on the bottom part is ramped linearly from $T_{a n}$ in the fore-section to $T_{c a t}$ at the beginning of the cathode, and is linearly decreased in the after-section down to $T_{a n}$. 


\section{c. Nondimensionalization}

The model discussed in the previous sections is made nondimensional by using a set of scales. The height $H$ between the electrodes is used to scale vertical distance, the length of the electrodes is used for scaling axial distance, and the applied voltage at the cathode is used for scaling potential.

An arbitrary number density $n_{0}$ is used to scale particle densities of the charged species. Neutral species are expressed as a mole fraction. The pressure and temperature at inlet stream are chosen to scale physical properties of gaseous species, which are used to define all dimensionless groups. Velocity is scaled by the ratio, $v_{\text {ref }}=$ $g(0.5 H)^{2} / \nu$, pressure is scated by $\mu_{\text {ref }} \nu_{\text {ref }} /(H / 2)$, and dimensionless temperature $\theta$ is defined as $T / T_{\text {ref }}$. The definitions of all the dimensionless parameters are shown in the Table I. Note that notation has not been changed from the dimensionless formulation.

The model of a dc glow discharge now takes the form

$$
\begin{aligned}
F o_{e} \frac{d n_{e}}{d t}+\frac{d j_{e}}{d z} & =\alpha j_{e} \\
F o_{p} \frac{d n_{p}}{d t}+\frac{d j_{p}}{d z} & =\beta \alpha j_{e} \\
\frac{d^{2} V}{d z^{2}} & =-b\left(n_{p}-n_{e}\right)
\end{aligned}
$$

where the dimensionless fluxes $j_{e}$ and $j_{p}$ are

$$
\begin{aligned}
& j_{e}=-\frac{d n_{e}}{d z}+P e_{e} n_{e} \frac{d V}{d z} \\
& j_{p}=-\frac{d n_{p}}{d z}-P e_{p} n_{p} \frac{d V}{d z}
\end{aligned}
$$

\begin{tabular}{|c|c|c|}
\hline Parameter & Definition & Value \\
\hline$\beta$ & $\frac{D_{e}}{D_{p}}$ & $2.3+10^{4}$ \\
\hline$\tau$ & $\min \left(\frac{(0 . S H)^{2}}{D}, \frac{(0.5 H)^{2}}{\mu_{1} V_{D C}}\right) ; i=e, p$ & $5.12 \cdot 10^{-9}(\mathrm{~s})$ \\
\hline$P e_{\mathrm{e}}$ & $\frac{\mu_{e} V_{D C}}{D_{e}}$ & $1.71 \cdot 10^{2}$ \\
\hline$P e_{p}$ & $\underline{\mu_{F}}{\underline{\mu_{D C}}}_{D}$ & $1.14 \cdot 10^{4}$ \\
\hline $\mathrm{Fo}_{\mathrm{e}}$ & $\frac{\left.(Q .5)^{p}\right)^{2}}{D \cdot T}$ & $1.71 \cdot 10^{2}$ \\
\hline$F o_{p}$ & $\frac{(0.5 H)^{2}}{D_{n} \tau}$ & $4.00 \cdot 10^{6}$ \\
\hline$\delta$ & $p k \xi(0.5 H)$ & 5.22 \\
\hline$\epsilon$ & $p a V_{D C}(0.5 H)$ & $1.48 \cdot 10^{2}$ \\
\hline$b$ & $\frac{n 0(0.5 H)^{2}|e|}{y_{0 r e 0}}$ & 12.06 \\
\hline$G r$ & $\rho_{r e f}^{2}(0,5 H)^{3} g$ & $3.12 \cdot 10^{-2}$ \\
\hline $\mathrm{Ra}$ & $G r \frac{\mu_{r e f}^{2}}{\mu_{r e f} C \rho_{r e S}}$ & $2.21 \cdot 10^{-2}$ \\
\hline $\mathrm{Pe}_{\mathrm{SiH}_{4}}$ & $\frac{(0 . S H)^{3} g \rho_{r e f}}{\mu_{r e f} D_{r e f}}$ & $2.68 \cdot 10^{-2}$ \\
\hline$k_{4}$ & $\begin{array}{l}\text { Reaction consiant Eq. (47) } \\
\text { Refor }\end{array}$ & $5.0 \cdot 10^{-18}\left(\mathrm{~m}^{3} \cdot \mathrm{s}^{-1}\right)$ \\
\hline$k_{5}$ & Reaction constant Eq. (48) & $1.0 \cdot 10^{-16}\left(\mathrm{~m}^{3} \cdot \mathrm{s}^{-1}\right)$ \\
\hline$k_{6}$ & Reacrion constant Eq.(49) & $1.7 \cdot 10^{-17}\left(\mathrm{~m}^{3} \cdot \mathrm{s}^{-1}\right)$ \\
\hline
\end{tabular}

TABLE I. Definitions of parameters and rypical values,
The Friedland equation is written as:

$$
\frac{d \alpha}{d z}+\alpha^{2}+\delta \alpha-\epsilon \frac{d V}{d z}=0
$$

1. Continuity equation

$$
\nabla \cdot\left(\frac{U}{\Theta}\right)=0
$$

\section{Momentum equations}

$$
\begin{aligned}
\frac{G r}{\Theta}\left(u_{y} \frac{\partial u_{y}}{\partial y}+u_{z} \frac{\partial u_{y}}{\partial z}\right)\left(\frac{L}{H}\right)= & -\left(\frac{H}{L}\right) \frac{\partial p}{\partial y} \\
& -\left(\frac{H}{L}\right) \frac{\partial \tau_{y y}}{\partial y}-\frac{\partial \tau_{y z}}{\partial z} \\
\left(\frac{G r}{\Theta}\right)\left(u_{y} \frac{\partial u_{z}}{\partial z}+u_{z} \frac{\partial u_{z}}{\partial z}\right)= & -\frac{\partial p}{\partial z}-\frac{\partial \tau_{z z}}{\partial z} \\
& -\left(\frac{H}{L}\right) \frac{\partial \tau_{y z}}{\partial y}+\frac{1}{\Theta}
\end{aligned}
$$

where

$$
\begin{gathered}
\tau_{y y}=-\left(\frac{\mu}{\mu_{r e f}}\right)\left(\frac{2}{3}\right)\left(2 \frac{\partial u_{y}}{\partial y}-\frac{\partial u_{z}}{\partial z}\right) \\
\tau_{z z}=-\left(\frac{\mu}{\mu_{r e f}}\right)\left(\frac{2}{3}\right)\left(2 \frac{\partial u_{z}}{\partial z}-\frac{\partial u_{y}}{\partial y}\right) \\
\tau_{y z}=-\left(\frac{\mu}{\mu_{r e f}}\right)\left[\left(\frac{H}{L}\right) \frac{\partial u_{z}}{\partial y}+\left(\frac{L}{H}\right) \frac{\partial u_{y}}{\partial z}\right]
\end{gathered}
$$

\section{Energy equation}

$$
\begin{aligned}
\left(\frac{R a}{\Theta}\right)\left(\frac{C_{P}}{C_{p_{r e f}}}\right)\left(u_{y} \frac{\partial \Theta}{\partial y}+u_{z} \frac{\partial \Theta}{\partial z}\right)= & \\
& \left(\frac{k}{k_{r e f}}\right)\left[\left(\frac{H}{L}\right)^{2} \frac{\partial^{2} \Theta}{\partial y^{2}}+\frac{\partial^{2} \Theta}{\partial z^{2}}\right]
\end{aligned}
$$

\section{Concentration equation}

$$
\begin{aligned}
\left(\frac{P e_{i m}}{\Theta}\right)\left(u_{y} \frac{\partial x_{i}}{\partial y}+u_{z} \frac{\partial x_{i}}{\partial z}\right)= & \frac{\partial}{\partial y}\left(-\frac{D_{i}}{D_{r e j} \Theta}\left(\frac{\partial x_{i}}{\partial y}+\frac{\alpha_{i} x_{i}}{\Theta} \frac{\partial \Theta}{\partial y}\right)\right) \\
+ & \left(\frac{H}{L}\right)^{2} \frac{\partial}{\partial z}\left(\frac{D_{i}}{D_{r e f} \Theta}\left(\frac{\partial x_{i}}{\partial z}+\frac{\alpha_{i} x_{i}}{\Theta} \frac{\partial \Theta}{\partial z}\right)\right) \\
\pm & D a f\left(\sum_{j} x_{j}\right)
\end{aligned}
$$




\section{NUMERICAL METHOD}

Based on our assumptions, the solving procedure has been divided into two parts: the glow discharge and the hydrodynamic description of the reactor. Both are coupled through the reaction between electrons and silane. Other interactions between plasma field and gas flow in the reactor are not taken into consideration.

\section{A. Glow discharge}

Due to high nonlinearity and strong coupling of the governing equations, the treatment of these equations poses a difficult numcrical problem. Examples of a few attempts to solve this problem can be found in the litcrature.

1. Ward ${ }^{11}$ pioneered numerical modeling of glow discharges. Hc used a shooting technique to solve the equations.

2. Lowke and Davies ${ }^{12}$ used an explicit relaxation method and obtained the steady state from the dynaınic form of the equations; a predictor-corrector method was used for time integration.

3. Graves and Jensen ${ }^{6}$ solved both the $\mathrm{dc}$ and if problem. The latter problem was considered as a boundary value problem in the time domain, and a Fouricr expansion was used to present time variations.

4. Kushner ${ }^{13}$ discretized spatial functions by finite differences and integrated the equations in time by a third order Runge-Kutta.

5. Boeuf ${ }^{14}$ adapted the implicit exponential scheme of Scharfetter and Gummel to model a two-dimensional dc glow discharge.

The problem of electron and hole transport in semiconductor devices is analogous to the glow discharge problem.

6. Traar et al. ${ }^{15}$ discussed a wide range of iteration methods for these types of convective dominant transport equations.

The implicit exponential scheme of Scharfetter and Gummel ${ }^{14}$ has been chosen to discretize the continuity equations. The main advantage of this scheme is its robustness, stability, and ability to deal with situations where either the convection or diffusion component of the total flux is dominant (these situations correspond, respectively, to the sheath and plasma regions).

The source term in the continuity cquation is implemented into a computer code as a continuous and smooth function of ionization coefficient $\alpha$, and electron flux $j_{e}$ :

$$
r_{i}=\left(\frac{1}{2}+\frac{1}{\pi} \tan ^{-1}(s * \alpha)\right) \alpha\left|j_{e}\right|
$$

and $s$ is a parameter chosen arbitrarily large.

The symmetric finite difference approximation is used for the Poisson equation. Friedland's equation is discretized by backward finite difference. Physical domain (cathode $=0$, anode $=H$ ) is mapped onto a computational domain (interval $x \in[-1,1]$ ). In our calculation an equidistant mesh is used.

After discretizing in the space domain, an initial value problem is obtained which is integrated by a second-order accurate trapezoid rule. The NewtonRaphson iterative scheme is used to obtain the solution at each stcp and finaily to find steady-state solution when the time derivatives diminish. The discretization of governing equations has resulted in the set of nonlinear algebraic-equations

$$
\Omega(y)=0
$$

where the vector of unknowns is represented as

$$
y=\left(n_{e 4 i-3}, V_{4 i-2}, n_{p 4 i-1}, \alpha_{4 i}, \ldots\right) \quad i=1,2, \ldots N_{t}
$$

$N_{t}$ : total number of nodes in the computational domain.

Since matrix $\Omega$ is sparse, the advantage of its bandmatrix representation is used for calculations. Each step in the Ncwton-Raphson method requires the solution of the lincar system. In the numcrical routine LUdecomposition has been used. At each step (starting from $i \geqslant 2$ ) the ratio $\left\|\delta_{i-1}\right\|_{\infty} /\left\|\delta_{i}\right\|_{\infty}$ is checked for convergence against the specified number and only if this ratio becomes smaller than 100 , the Jacobian is updated; otherwise the LU-decomposition from the previous step is used. The electric field and ionization term are updated only once at each time step.

During marching in time the size of the time step depends upon deviation from steady state solution. The strategy for varying the step size $\Delta t_{n}$ is based on estimation of the local truncation error. ${ }^{16}$

\section{B. Numerical solution of hydrodynamic model}

The partial differential equations conscrving momentum, heat, and mass transfer are numerically solved by the Galerkin finite element method. The code was developed to handle both diffusion and advectiondominated problems. In order to overcome oscillations in the Jatter case, artificial dispersion is introduced. ${ }^{17}$

In applying the Galerkin finite element method to the hydrodynamic model, pressure has to be approximated with trial functions of one order lower than the trial functions for velocity, temperature, and concentration. The artificial dispersion is introduced into the momentum equations in a form that is referred to as the streamlinc upwind method (SU) ${ }^{17}$ and into the energy and concentration balances by the SU Pctrov-Galerkin method (see Brooks and Hughes ${ }^{18}$ ). Finally, the system of nonlinear algebraic equations is solved by the Newton-Raphson method. To improve the initial guess, a false transient method is adopted. 


\section{RATE OF SILANE DISSOCIATION}

Although the glow discharge deposition of silane to produce a-Si:H is the most studied plasma enhanced CVD process, the details of the silane plasma chemistry are still debated. Controversy exists over the dominance of the silylene or silyl radicals in the deposition process. ${ }^{5,19}$

In pure amorphous silicon, atoms that are only threefold coordinated have an unpaired electron, referred to as a dangling bond. These unpaired electrons impair the film's photoconductivity and photoluminescence. In hydrogenated amorphous silicon the density of dangling bonds is greatly reduced, because hydrogen can bond to single silicon atoms. Not all hydrogen incorporated in the silicon structure attaches to dangling bonds; it is reported by Thomas ${ }^{20}$ that hydrogen preferentially diffused into a hydrogen-rich phase that was interdispersed between a columnar phase of monohydride $\mathrm{Si}-\mathrm{H}$ groups. Only hydrogen that is incorporated in the latter phase will lead to a reduction in the dangling bond density. In a weakly ionized glow, neutral radical species in the ground electronic state play important roles in depositing $\mathrm{a}-\mathrm{Si}: \mathrm{H}$; they directly participate in the chemical and physical processes on the surface of the deposit.

\section{Plasma chemistry}

Electron collisions with silane produce a wide range of products, but the majority of these products are not stable. The major branching processes for neutral dissociation are:

$$
\begin{aligned}
& e+\mathrm{SiH}_{4} \stackrel{k_{1}}{\longrightarrow} \mathrm{SiH}_{3}+\mathrm{H}+e \\
& \stackrel{k_{2}}{\longrightarrow} \mathrm{SiH}_{2}+\mathrm{H}+\mathrm{H}+e \\
& \stackrel{k_{3}}{\longrightarrow} \mathrm{SiH}_{2}+\mathrm{H}_{2}+e
\end{aligned}
$$

We define the following branching ratios:

$$
\begin{aligned}
& \alpha=\frac{k_{1}}{k_{1}+k_{2}+k_{3}} \\
& \beta=\frac{k_{2}}{k_{1}+k_{2}+k_{3}}
\end{aligned}
$$

Generation of $\mathrm{H}$ atoms during electron impact dissociation effectively increases the rate of production of radicals due to the following reaction:

$$
\mathrm{H}+\mathrm{SiH}_{4} \stackrel{\dot{k}_{4}}{\rightarrow} \mathrm{SiH}_{3}+\mathrm{H}_{2}
$$

Silylene is depleted by an insertion reaction with silane

$$
\mathrm{SiH}_{2}+\mathrm{SiH}_{4} \stackrel{\kappa=}{\longrightarrow} \mathrm{Si}_{2} \mathrm{H}_{6}
$$

The insertion reaction is potentially fast and it produces disilane, which does not incorporate into the growing film. The silyl radical is more stable than the silylene radical and participates in few depleting reactions. The most important of these is a recombination reaction:

$$
\mathrm{SiH}_{3}+\mathrm{SiH}_{3} \stackrel{k_{6}}{\longrightarrow} \mathrm{SiH}_{2}+\mathrm{SiH}_{4}
$$

Kushner ${ }^{5}$ undertook a detail modeling of the silane dissociation chemistry in a glow discharge. He used maximum and minimum values for the rate constants of Eq. (48) and (49) which were available in literature. It was also found that the deposition rate is most sensitive to the yield of $\mathrm{H}$ atoms from the dissociation to $\mathrm{SiH}_{2}$. Silyl is the only main contributor to film growth when the sticking coefficient exceeds 0.5 . Along the guidelines provided by Kushner, ${ }^{5}$ the rate constants for reactions (47)-(49) are listed in Table I.

ltabashi ${ }^{21}$ et al. measured (and apparently the first time to do so) the spatial distribution of silyl radicals in an rf silane plasma, using infrared diode laser absorption spectroscopy. They used the following rate of deposition expression:

$$
\begin{aligned}
R_{d} & =\Gamma \times S \times \frac{m}{p} \\
\Gamma & =\frac{D_{e}}{\rho \times \beta} \frac{d\left(\mathrm{SiH}_{3}\right)}{d x}
\end{aligned}
$$

The loss probability $\beta \approx 0.26$, the sticking coefficient $S=0.09$, which is quite a low value compared to the threshold value of Kushner, ${ }^{5} m=4.7 \times 10^{-23} \mathrm{~g}$, and $\rho-2.21 \mathrm{~g} / \mathrm{cm}^{3}$. They calculated $R_{d}=0.40 \mu \mathrm{m} / \mathrm{h}$ for a $\mathrm{SiH}_{4} / \mathrm{H}_{2}$ plasma al $80 \mathrm{mTorr}$ and $125 \mathrm{~W}$ power deposition between a $3 \mathrm{~cm}$ gap. The experimental rate was $0.64 \mu \mathrm{m} / \mathrm{h}$. To distinguish properly between silyl and silylene, their respective collision cross sections are required; unfortunately only global cross sections are available. Perrin et $a l^{22}$ measured global cross sections for dissociation of methane and silane for electron energies between 8 and $110 \mathrm{eV}$. The former value is the experimentally found threshold energy for dissociation of silane. Vicek ${ }^{10}$ used explicit forms of cross sections for $\mathrm{Ar}$, and we used their functional form to fit l'errin's experimental results.

$$
\sigma_{D}=\left(7.1638 \times 10^{-10}\right)^{2} \frac{\left(\frac{\varepsilon}{8}-1\right)}{\left(\frac{\epsilon}{8}\right)^{2}} \ln \left(1.04315 \times \frac{\epsilon}{8}\right)
$$

This curve fits the measured range of electron energy very well, and we used it to evaluate the cross section for the dissociation kinetics. To find the rate constant for dissociation, one requires knowledge of the electron 
energy distribution function (EEDF):

$$
k_{D}=\int_{8}^{\infty}\left(\frac{2 \epsilon}{m}\right)^{0.5} f(\bar{\epsilon}, \epsilon) \sigma_{D}(\epsilon) d \epsilon
$$

where $\bar{\epsilon}(x)$ denotes an average clectron energy at $x$.

The mean electron energy will be determined for the plasma, using the Friedland equation to model ionization. In Fig. 2 normalized values of the mean electron energy and the dissociation rate constants are shown. A sharp increase in energy in the sheath region is followed by a decline to a low energy in the remainder of the plasma. The maximum energy for this example (i.e., $600 \mathrm{~V}$ over a $2 \mathrm{~cm}$ gap) is $110 \mathrm{eV}$. One should not mistakcnly attribute the decline in mean encrgy to collision processes. The main reason for the decrease in mean energy is the formation of an avalanche of new electrons, which gain far lcss encrgy than sheath electrons, and the mean is shifted toward this bulk of low-energy clectrons. Information on the sheath electrons is lost. Another important factor that we want to point out is the contribution of electrons formed in the sheath to the total flux of electrons. Although the sheath electrons form a small fraction of the total clectron number density, they form a significant fraction of the total electron flux. Therefore they makc an important contribution to the production of radical species.

The Maxwell distribution gives the highest $k_{D}$ value locally, but the values decrease sharply away from this point. The Druyvestcyn distribution gives a similar profile, but the values for $k_{D}$ atc smaller. The relation between reaction rate and mean cnergy is also evident from Fig. 2 and one can immediately see that most of the radical production will take place near the cathode. This conclusion is confirmed by the model of Yamaguchi

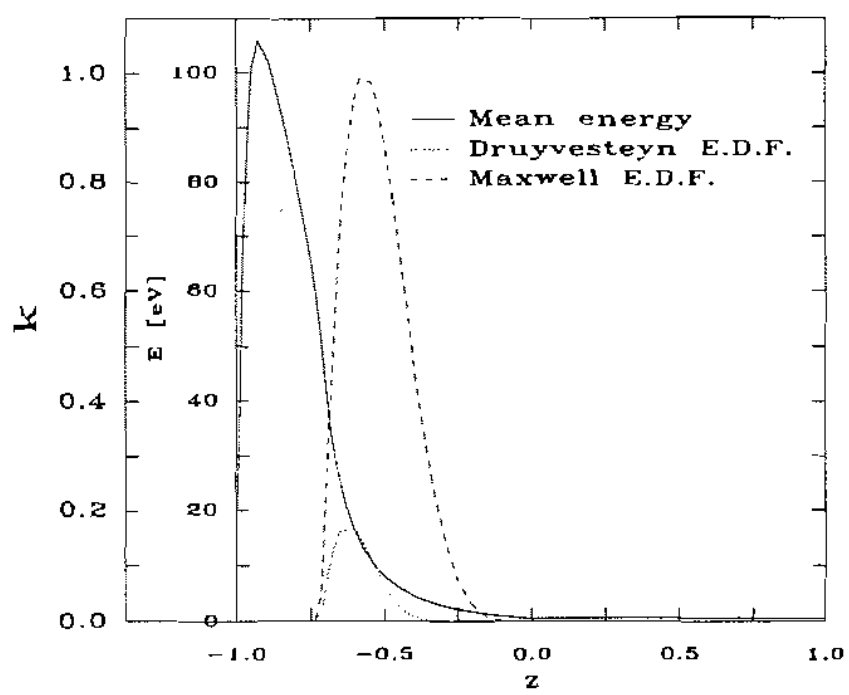

FIG. 2. Space evolution of electrons mean energy. Space evolution of reaction rate for Maxwell and Druyvesteyn electron energy distribution functions. et $a .^{23}$ where it was found that the silyl concentration reaches a maximum in a small region near the cathode. They used dissociation collision frequencies $R_{d}$ which were published by Ohmori et al. ${ }^{24}$

\section{RESULTS}

In this section the results of the plasma reactor model will be reported. But first a brief exposition of this section will be helpful. We start off by defining a scalar norm of the uniformity of deposition to measure reactor performance us a function of different parameters. Some results of the glow discharge itself are presented. Charged species concentrations, potential, and ionization rates are shown for a range of applied voltages, distances across the gap, and the total pressure.

For the sake of comparison, a base case is defined. The concentration fields of all species are shown for the base case. Next we do a sensitivity analysis. All the design and control variables are changed by $\pm 10 \%$ from their basc values and the change in the norm is calculated. Deposition rate profiles along the gap arc shown for these different parameter values, including a comparison betwcen the Maxwell and Druyvesteyn distributions. The effects of branching ratios (silanc dissociation) on the deposition rate profiles are also prescnted.

It is not easy to define an unambiguous norm for a reactor. Solid state physical requirements of the film will most likely dictate different criteria. However, we will assume that only two factors determine the performance of the reactor: the rate of deposition and the uniformity of deposition. The first consideration is bounded from below by economical factors and from above by physical factors (e.g., particle formation, film quality, etc.). We will report average deposition rates on both electrodes, and whether it meets the economical and physical criteria is not addressed in this work. The following norm is proposed to measurc the uniformity of deposition, and it will be used to compare the effects of different parameters on the system.

$$
\|\cdot\|=\frac{1}{L} \int_{0}^{L}\left[1-j_{d} / \overline{j_{d}}\right]^{2} d y
$$

wherc $j_{d}$ is the sum of the silyl and silylene fluxes at the cathode/anode and $\overline{j_{d}}$ is the averaged value. It's obvious that the smaller the norm, the better the uniformity.

\section{A. Results of glow discharge}

The glow discharge reactor, as was outlined in the model description, conforms to a parallel-plate configuration and it is also consistent with some of the reactors 

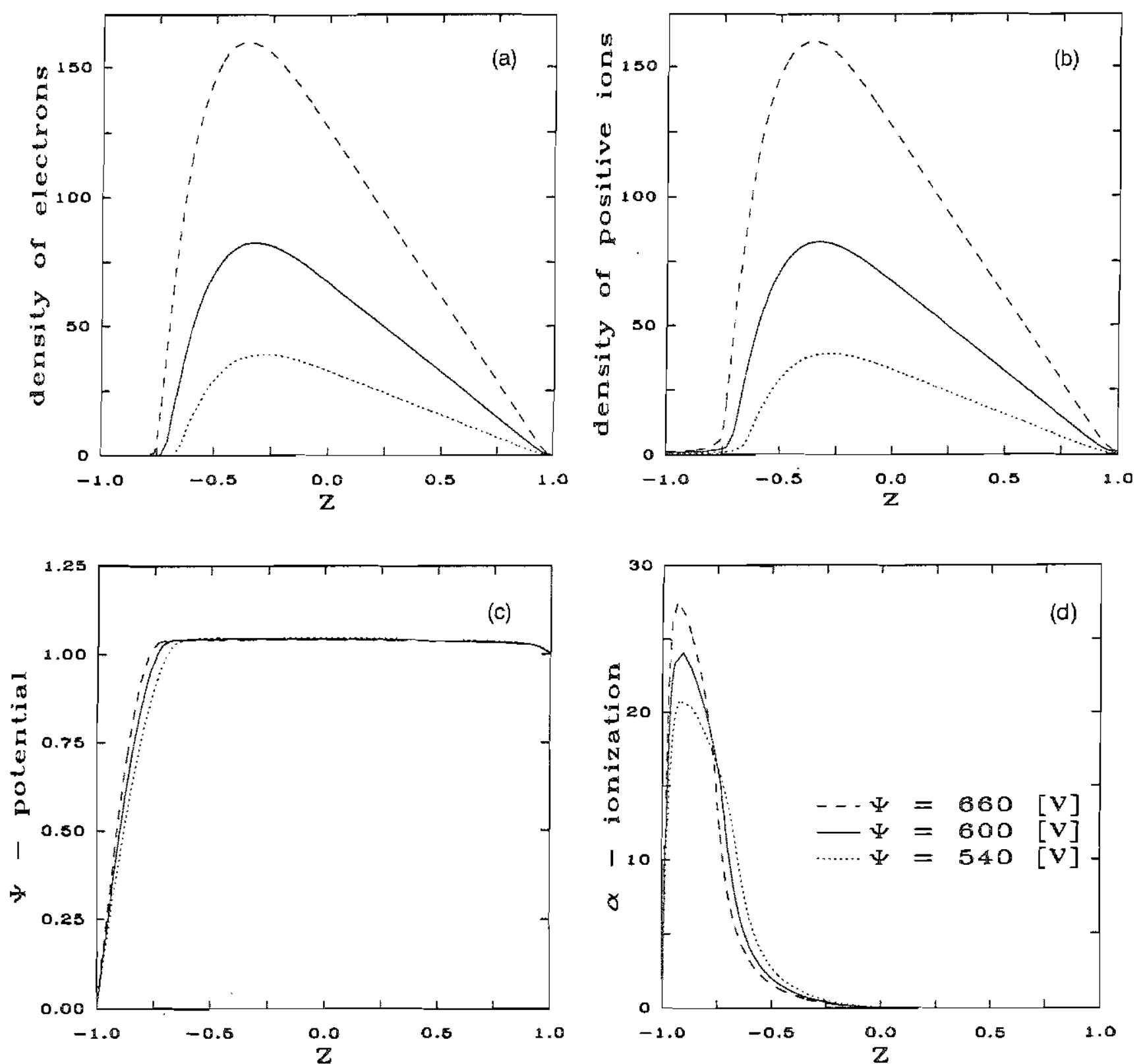

FIG. 3. Space evolution of glow discharge plasma lor different voltages $V=600,540$, and $660 \mathrm{~V}$ : (a) concentration of electrons $\left(\times 10^{15} / \mathrm{m}^{3}\right)$, (b) concentration of positive ions $\left(\times 10^{15} / \mathrm{m}^{3}\right)$, (c) potential, and (d) ionization rate $(1 / \mathrm{cm})$.

in use at the "Sunshine Project" in Japan." A base pressure of 1.5 Torr was used and the gas, containing $1 \% \mathrm{SiH}_{4}$, was fed at a rate of $10 \mathrm{~cm} / \mathrm{s}$. Figure 3 is a compounded presentation of the results for a $2 \mathrm{~cm}$ gap between the electrodes and for different voltages. For the base case of $600 \mathrm{~V}$, the sheath thickness is approximately $14 \%$ of the gap space and it decreases to $12 \%$ when the voltage is increased by $60 \mathrm{~V}$. The increase in potential decreases the width of the sheath and the electron density has increased to $164 \times 10^{15} / \mathrm{m}^{3}$. In terms of reactor performance, these changes will lead to an inerease in silyl deposition for the following reasons.
A decrease in sheath thickness leads to a decrease in the distance silyl has to diffuse. A higher electron density, in a stronger electric field, will increase the formation of radical species. Lowering the voltage by $10 \%$ leads to a sheath thickness of $\approx 18 \%$ and the electron density drops to $39 \times 10^{15} / \mathrm{m}^{3}$. The qualitative changes in the piasma when changing the potential are clear: increasing the potential leads to a narrower sheath and a higher electron density, electron energy increases, and silyl production goes up.

The maximum number densities of the charged species quadrupled from the lower to the higher voltage. 
The potential shows little change, but a slight shift in the sheath region is noticeable. The potential increases sharply across the sheath, and is quite hat in the plasma region. A small drop in potential is noticeable near the anode. The markedly different electric field near the cathode compared to the anodc will lead to quite an intense ion bombardment at the cathode and to a much lesser extent at the anode. The film's quality is infuenced by the ion bombardment and a higher density film can be expected on the cathode. The ionization rate is limited to the sheath region, and the maximum increases with increased voltage, as expected. Figure 4 shows the resuits for $600 \mathrm{~V}$, but $\pm 10 \%$ changes in pressure. The system shows less sensitivity to these changes; e.g., the maximum number densities changed $\approx 80 \%$ between the extrema. Similar results are obtained when the gap distance is changed. The reader is cautioned in interpreting the results in Fig. 5 since the gap space was nondimensionalized by different values of $H$. The discharge exhibits even lesser sensitivity than in the previous two cases. Finally, Fig. 6 shows results for different mean temperatures in the gap. Apart from changing the density, temperature also affects the parameters in the Friedland formulation and the transport parameters (cf. Appendix). An important point that stays in neglect is the temperature dependency of the secondary emission coefficient. Also note that any thermionic emission is not considered in this model.

\section{B. Plasma chemistry results}

To solve for the concentration fields, one requires knowledge of the kinetics for $\mathrm{SiH}_{4}$ dissociation. A full section is dedicated to this problem, because it is the part of the system onc has to understand, and has to control, if one really wants to benefit from a mathematical model. Any improvement in the system is possible only if the kinetics of the dissociation reaction is improved. Tracking back this line of reasoning, it follows that the EEDF plays a very important role. The EEDF is an output of the glow discharge and it can be controlled by changing the potential and pressure (these are the most convenicnt control variables).

In Figs. 7-12 isolines of the molar fractions are shown for $\mathrm{SiH}_{4}, \mathrm{SiH}_{3}, \mathrm{SiH}_{2}, \mathrm{Si}_{2} \mathrm{H}_{6}, \mathrm{H}_{2}$, and $\mathrm{H}$, respectively. Silane depletion is highest near the cathode, as can also be observed from the maxima in silyl and silylene concentrations in this region. The high diffusivities of the radical species lead to their presence well outside the reactor zone. However, therc is a significant difference in the distribution of silyl and silylene. The latter species is aIso depleted by an insertion reaction with silane and thus silylene is located only near the cathode (where silane is least) whilc silyl is also present near the anode. The high mobilities of molecular and atomic hydrogen are reflected in the relatively large concentrations outside the reactor zone, both up and downstream. The flux of silyl and silylene to the cathode and anode can now be determincd $\left(j_{i}, i=\mathrm{SiH}_{3}, \mathrm{SiH}_{2}\right)$ :

$$
j_{i}=C D_{i}\left(\frac{d x_{i}}{d x}+\alpha_{i} x_{i} \frac{d \ln (T)}{d x}\right) .
$$

The rate of deposition is calculated in the same way as Yamaguchi et al. ${ }^{23}$ it is expressed in unit of $\mu \mathrm{m} / \mathrm{h}$.

$$
R_{d}=\frac{S}{\rho}\left[j_{\mathrm{SiH}_{3}}+j_{\mathrm{SiH}_{2}}\right] \times 3.6 \times 10^{9} .
$$

and $\rho=4.29 \times 10^{28}$. The sticking coefficient is taken as $S=1$. In the following section, deposition rates and profiles are shown at different operating conditions and for different clectron encrgy distributions.

\section{Deposition}

To analyze the importance of the electron energy distribution, we used the following two distributions:

$$
\begin{aligned}
\operatorname{EEDF}_{\text {Mwel }} & =\frac{4}{\pi^{0.5}} \times \frac{\epsilon}{1.128^{2} \bar{\epsilon}^{2}} e^{-\epsilon / 1.128^{2} \bar{\epsilon}} \\
\operatorname{EEDF}_{\text {Dryy }} & =\frac{1.04 \epsilon^{0.5}}{\bar{\epsilon}^{1.5}} e^{-0.55(\epsilon / \bar{\epsilon})^{2}}
\end{aligned}
$$

In Figs. 13(a) and 13(b) the deposition profiles at the cathode are shown for the Maxwell and Druyvesteyn distributions. The rest of the conditions are consistent with the base case. The mean rate drops from $7.36 \mu \mathrm{m} / \mathrm{h}$ for the Maxwell distribution to $5.76 \mu \mathrm{m} / \mathrm{h}$ for the Druyvesteyn distribution. In both cases, silyl is the dominant depositing species. The results for the anode are shown in Figs. 14(a) and 14(b). Note the absence of silylene in this case. The mean rate now drops from $0.88 \mu \mathrm{m} / \mathrm{h}$ for the Maxwell distribution to $0.57 \mu \mathrm{m} / \mathrm{h}$ for the Druyvesteyn distribution.

The effects of branching ratios are presented in Figs. 13(a), 13(c), and 13(d) and Figs. 14(a), 14(c), and 14(d) for the cathode and anode. respectively, and also in Table II and Table III. Increasing $\beta$ leads to an increasc in deposition rate on both electrodes in the order of $0.3-0.5 \mu \mathrm{m} / \mathrm{h}$, and the same effect holds for an increase in $\alpha$. Another interesting result is the prescnce of a local maximum in the deposition profiles on the cathode, but the profiles on the anode are monotone. Referring to Fig. 8 the contours are converging along the channel, while they are slightly diverging near the cathode; this explains the difference in the deposition profiles.

In Fig. 15 and Fig. 16 we present the deposition profiles on the cathode and the anode, respectively, for diffcrent parameters. For the cathode in particular, the applied voltage proves to be the most sensitive parameter, and $\pm 10 \%$ changes in the base valuc results 

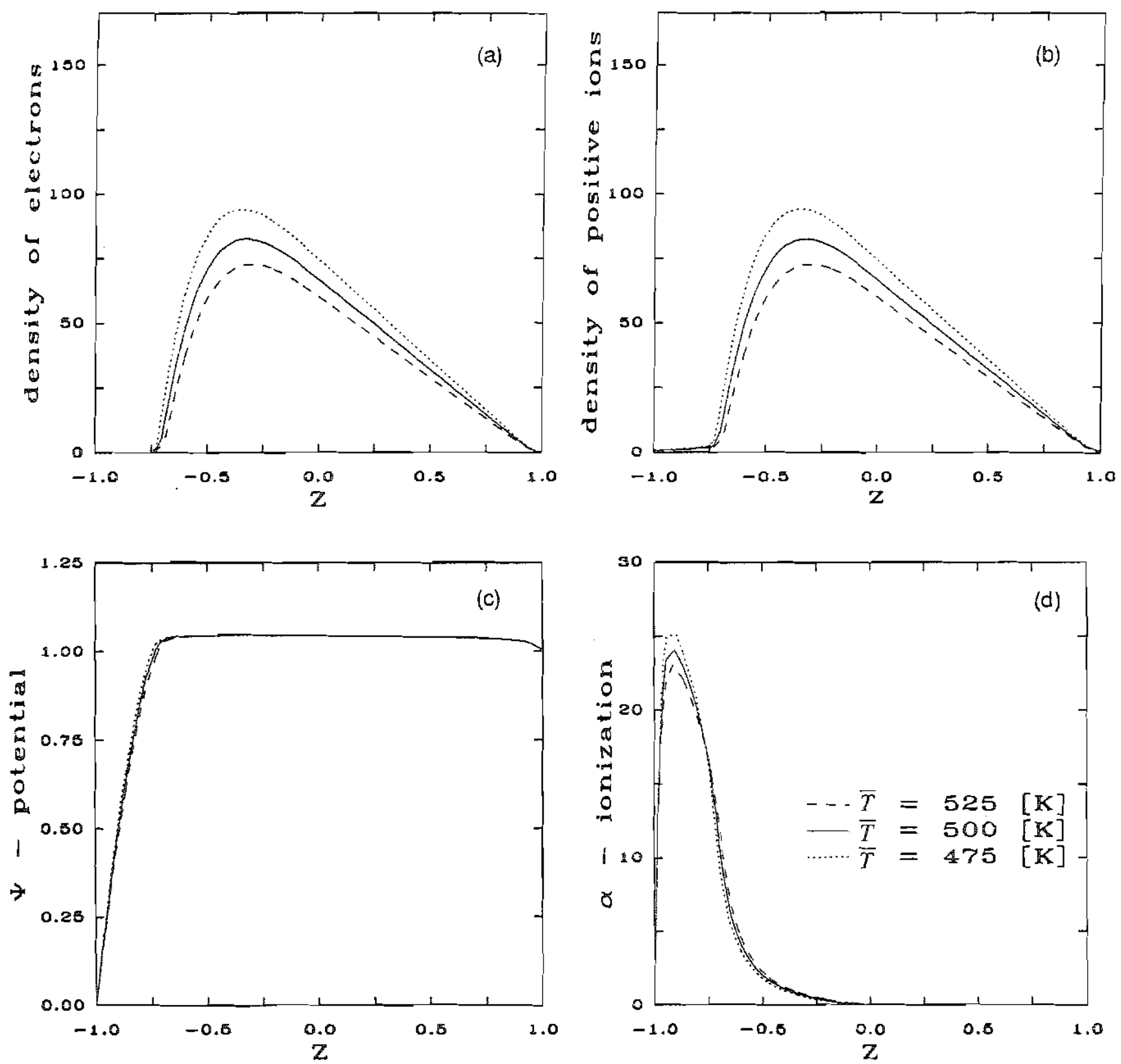

FIG. 6. Space evolution of glow discharge plasma for different gas temperatures in the reactor $\bar{T}=500,475$, and $525 \mathrm{~K}$ : (a) concentration of electrons, (b) concentration of positive ions, (c) polential, and (d) ionization ratc.

importance of controlling the energy and density of the electrons in the first place.

Increasing $E / p$ (higher voltage, lower pressure) and increasing the mean free path for electrons will lead to an increase in the high energy tail of the EEDF, and consequently in the production of radicals. The EEDF can also be altered by changing the composition of the gas. However, the collision cross section for different species depends nonlinearly on the energy of the electrons and the outcome is not always predictable.

The radical concentrations can aiso be changed by the introduction of radical scavengers (such as $\mathrm{NO}$ ), while the addition of hydrogen in Ar glow discharges lowers the mean electron energy. In chlorosilane plasmas this quenching effect of hydrogen inhibits the electron impact dissociation of $\mathrm{SiCl}_{4}$, increasing the free radical concentration in the plasma. It was experimentally observed that hydrogen addition can lead to one order of magnitude increase in free radicals. The relative weight for the radical-neutral reactions can be controlled by changing the free mean path for radicals and the residence time in the reactor. Flow speed, reactor length, and pressure are the relevant control and design variables. Control of the extraction rates for neutral radicals 


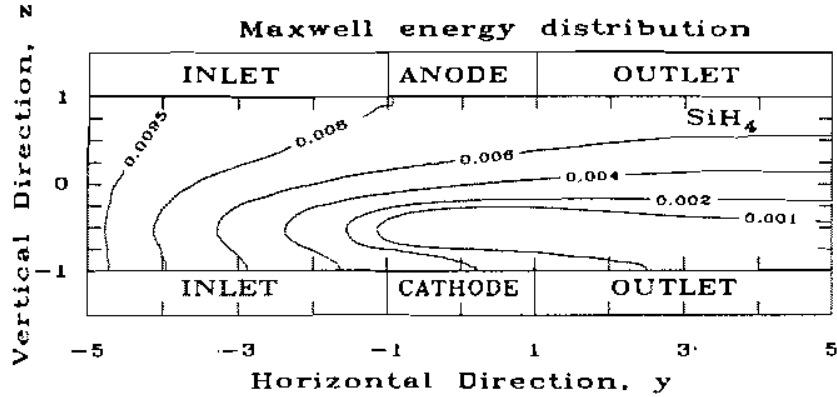

FIG. 7. Isolines of $\mathrm{SiH}_{4}$ molar fraction in the reactor.

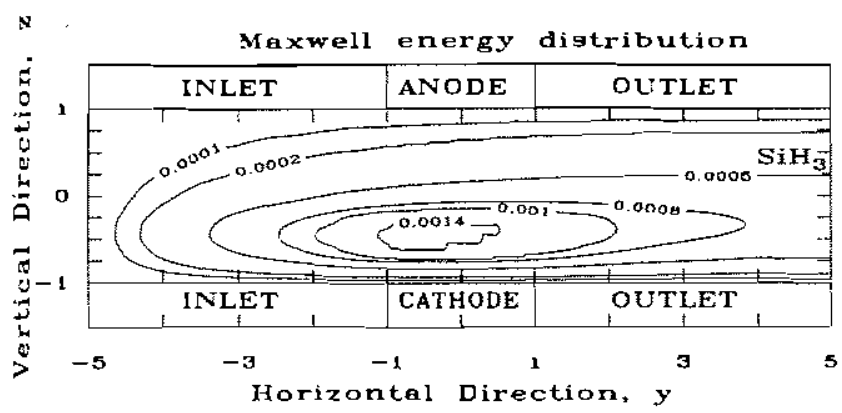

FIG. 8. Isolines of $\mathrm{SiH}_{3}$ molar fraction in the reactor.

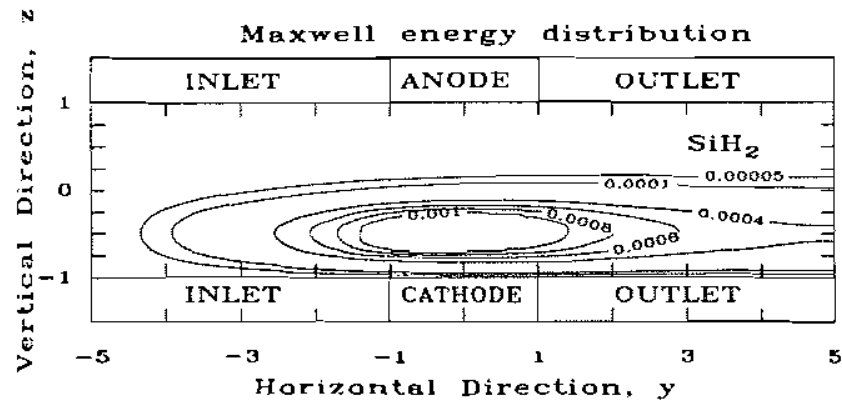

FIG. 9. Isolines of $\mathrm{Sil}_{2}$ molar fraction in the reactor.

is possible through the modification of their spatial distributions.

External deposition is becoming increasingly important, especially for applications that are sensitive toward ion bombardment. One approach is to inject the molecular gas (e.g., $\mathrm{SiH}_{4}$ ) into the after section and to place the substrate at the end of the after section. Enhanced deposition can be obtained in a stagnation point flow configuration.

Another important factor is the ion bombardment of the substrate surface. Film properties are modified by the ion bombardment. Ion-neutral interactions near the cathode can significantly influence this bombardment. Charge exchange wili lead to a decrease in the energy of the bombarding ions, but it can be quantified only by adapting the present model.

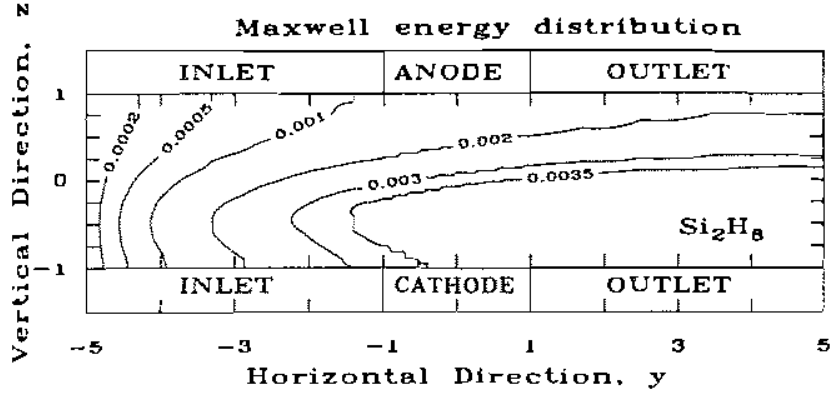

FIG. 10. Isolines of $\mathrm{Si}_{2} \mathrm{H}_{6}$ molar fraction in the reactor.

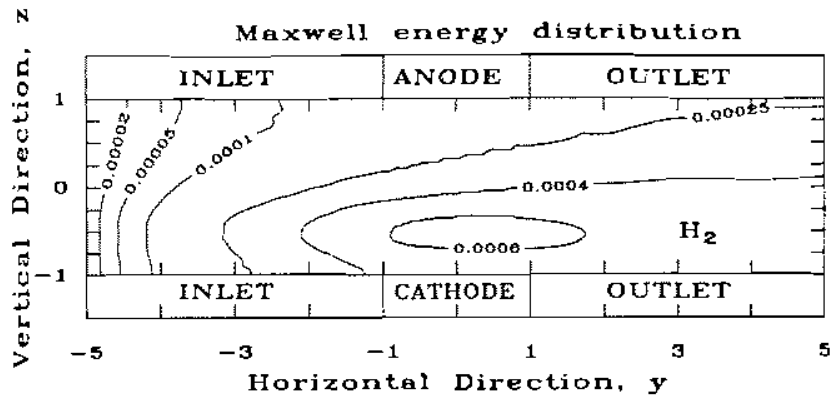

FIG. 11. Isolines of $\mathrm{H}_{2}$ molar fraction in the reactor.

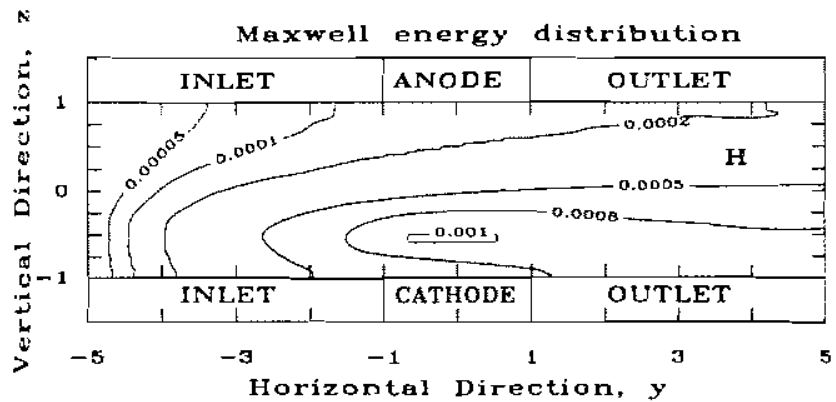

FIG. 12. Isolines of $\mathrm{H}$ molar fraction in the reactor.

\section{NOMENCLATURE}

a lonization collision efficiency

$$
\left(\mathrm{V}^{-1} \cdot \mathrm{m}^{-1} \cdot \text { Torr }^{-1}\right)
$$

$b \quad$ Dimensionless parameter; see Table 1

$C$ Concentration of feed gas (mole $\cdot \mathrm{m}^{-3}$ )

$C_{p} \quad$ Specific heat capacity $\left(\mathrm{J} \cdot \mathrm{kg}^{-1} \cdot \mathrm{K}^{-1}\right)$

$D_{i} \quad$ Diffusion coefficient of $i^{\prime}$ th species $\left(\mathrm{m}^{2} \cdot \mathrm{s}^{-1}\right)$

$E \quad$ Electric field $\left(\mathrm{V} \cdot \mathrm{m}^{-1}\right)$

$e \quad$ Unit charge (Coulomb)

$\mathrm{EEDF}_{i}$ Electron energy distribution function, $i=$ Maxwell, Druyvesteyn

Fo Fourier number

g Gravity constant $\left(\mathrm{m} \cdot \mathrm{s}^{-2}\right)$

$H \quad$ Gap space between electrodes (m) 
$j_{i} \quad$ Flux of $i$ th species $\left(\mathrm{m}^{2} \cdot \mathrm{s}^{-1}\right)$

$k \quad$ Total inelastic collision efficiency $\left(\mathrm{V}^{-1} \cdot \mathrm{m}^{-1} \cdot\right.$ Torr $\left.^{-1}\right)$

k Conductivity tensor $\left(\mathrm{W} \cdot \mathrm{m}^{1} \cdot \mathrm{K}^{-1}\right)$

$\mathrm{k}_{\mathrm{B}} \quad$ Boltzmann constant, $8.61727 \times 10^{-5}\left(\mathrm{eV} \cdot \mathrm{deg}^{-1}\right)$

$k_{D} \quad$ Rate constant for silane dissociation $\left(\mathrm{s}^{-1}\right)$

$k_{i} \quad$ Reaction rate constant $\left(\mathrm{m}^{3} \cdot \mathrm{s}^{-1}\right)$

$l_{D} \quad$ Debye length $(\AA)$

$n_{i} \quad$ Number density of $i^{\prime}$ th species $\left(\mathrm{m}^{-3}\right)$

$N \quad$ Total number of molecules

$p \quad$ Pressure in the system (Torr)

$\mathrm{Pe}_{i} \quad$ Peclet number of $i$ 'th species

$\mathrm{R}_{\mathrm{g}} \quad$ Cniversal gas constant $\left(\mathrm{J} \cdot \mathrm{mole}^{-1} \cdot \mathrm{K}^{-1}\right.$ )

Ra Raleigh number; see Table I

$R_{d} \quad$ Rate of deposition $\left(\mu \mathrm{m} \cdot \mathrm{h}^{-1}\right)$

$T$ Temperature of neutral and positive ions (K)

$T_{e} \quad$ Electron temperature $(\mathrm{eV})$ or $(\mathrm{K})$

$t$ Time (s)

U Velocity vector of neutral species $\left(m \cdot s^{-1}\right)$

$V \quad$ Potential (V)

$x_{i} \quad$ Mole fraction of $i$ 'th species

$y \quad$ Space variable, along length of reactor $(\mathrm{m})$

$z \quad$ Space variable, between electrodes $(m)$

\section{Greek symbols}

$\alpha \quad$ Townsend primary ionization coefficient $\left(\mathrm{m}^{-1}\right)$

$\alpha_{i} \quad$ Thermal diffusion coefficient of $i$ 'th species

$\beta \quad$ Dimensionless parameter; see Table 1

$\delta \quad$ Dimensionless parameter; see Table I

$\epsilon \quad$ Dimensionless parameter; see Table 1, also used as electron energy (eV)

$\epsilon_{0} \quad$ Permittivity of frec space, $8.85 \times 10^{-12}\left(\mathrm{C} \cdot \mathrm{V}^{-1} \cdot \mathrm{m}^{-1}\right)$

$\rho \quad$ Density of gas phase $\left(\mathrm{kg} \cdot \mathrm{m}^{-3}\right)$

$\mu \quad$ Viscosity of neutral species $(\mathrm{Pa} \cdot \mathrm{s})$

$\mu_{i} \quad$ Mobility of $i^{\prime}$ th species $\left(\mathrm{m}^{2} \cdot \mathrm{V}^{-1} \cdot \mathrm{s}^{-1}\right)$

$\xi \quad$ Energy, for activation or ionization (cV)

$\sigma_{i} \quad$ Collision cross section for $i^{\prime}$ th process $\left(\mathrm{m}^{2}\right)$

\section{Subscripts}

$\begin{array}{ll}\text { an } & \text { Anode } \\ c a t & \text { Cathode } \\ D & \text { Dissociation } \\ e & \text { Electron } \\ p & \text { Positive ion } \\ \text { ref } & \text { Reference value }\end{array}$

\section{REFERENCES}

1. A. Inspektor-Koren, Surf. and Coat. Technol. 33, 31 (1987).
2. J. D. Joannopoulos and G. Lucovsky, The physics of Hydrogenated Amorphous Silicon I (Springer-Verlag. Berliu, 1984).

3. S. Nonaka, Jpn. J. Appl. Phys. 29, 571 (1990).

4. J. M. Thomis, Properties of Amorphous Silicon (INSPEC, New York, 1985).

5. M. J. Kushner, J. Appl. Phys 62, 2803 (1987).

6. D. Graves and K. F. Jensen, IEEE Trans. Plasma Sci. PS-14, 78 (1986).

7. P. Segur, M. Yousfi, J P. Bucuf, E. Marode, A.J. Davies, and J.G. Evans, Microseopic Treatment of non-Equilibrium Regions, Proc. 11th Cont. on Plasma (1982).

8. L. Friedland, J. Phys. D: Appl. Phys. 7, 2246 (1974).

\$. L. Fricdland and Yu M. Kagan, J. Phys. D: Appl. Phys. 19, 1019 (1980).

10. J. Vicek, J. Phys. D: Appl Phys. 22, 623 (1988).

11. A. L. Ward, J. Appl. Phys, 33, 2789 (1962).

12. J. J. Lowke and D. K. Divies, J. Appl. Phys. 48, 499) (1977).

13. M.J. Kushner, J. Appl. Phys. 63 (8), 2532 (1988).

14. J-P. Boeuf, J. Appl. Phys. 63 (1988).

15. K. P. Traar, W. Mader, O. Heineichsberger, and S. Selberherr, in Supercompuing 90 (IEEE Comp. Press).

16. P. M. Gresho, R. L. Lec, and R. L. Sani, in Recent Advances in Numerical Methods, Fluids, edited by $C$. Taylor and K. Morgan (Pineridge Press, Swansea), Vol. 1, pp. 22-79.

17. T.J. R. Hughes and A.N. Brooks, in Finite Element Methods for Converive Dominated Flows (AMD 34, ASME, New York, 1979).

18. A. N. Brooks and T.J.R. Hughes, Comp. Meth. Mech., 32 (1982).

19. P.M. Martincau and P. B. Davies, Chem. Brit. Ocl., 1018 (1989).

20. J. M. Thomas, in Propertics of Amorphous Silicon (INSPEC Now York, 1985)

21. N. Llabashi, N. Nishiwaki, M. Magane, S. Naito, T. Golo, A. Matsuda, C. Yamadia, and E. Hirota, Jpn. J. Appl. Phys. 29, L505 (1990).

22. J. Perrin, J. P. M. Schmitt, G. DeRosny, B. Drevillon, J. Huc, and A. Llorel, Chem. Phys. 73, 383 (1982).

23. Y. Yamaguchi, A. Sumiyama, R. Hatlori, Y. Morokuma, and T. Makabe, J. Phys. D: Appl. Phys. 22, 505 (1989).

24. Y. Ohmori, M. Shimozuma, and H. Tagashira, J. Phys. D: Appl. Phys. 19. 1029 (1986).

25. K. Tichibana, Pure and Appl. Chem. 60, 769 (1988).

26. E. W. McDaniel and E. A. Mason, The Mobility and Diffision of Ions in Gases (John Wilky and Sons, New York, 197.3).

27. S-K. Park and D.J. Economou. J. Appl. Phys. 68, 3904 (1990).

28. A. A. Kruithof and F.M. Penning, Plhysics 4, 430 (1937).

24. M. Suzuki, T. Taniguchi, and H. Tagashira, J. Pliys, D: Appl. Phys. 23, 842 (1990).

30. H. Ltoh, M. Kawaguchi, M. Takada, Y. Nakalo, and H. Tagatshira, J. Plyys. D: Appl. Phys. 22, 1095 (1989).

31. R.J. Carman, J. Plyys. D: Appl. Phys. 22, 55 (1989)

\section{APPENDIX: PHYSICAL PROPERTIES}

The correctness of a mudeling effort is detcrmined to a large extent by the accuracy and availability of reliable physical data. The physical properties of the glow discharge are first discussed and then the properties that are required in the hydrodynamic model are presented. Complete references to the source of the data are given. When the property was determined by a corretation, the mothod is briefly oullined. 

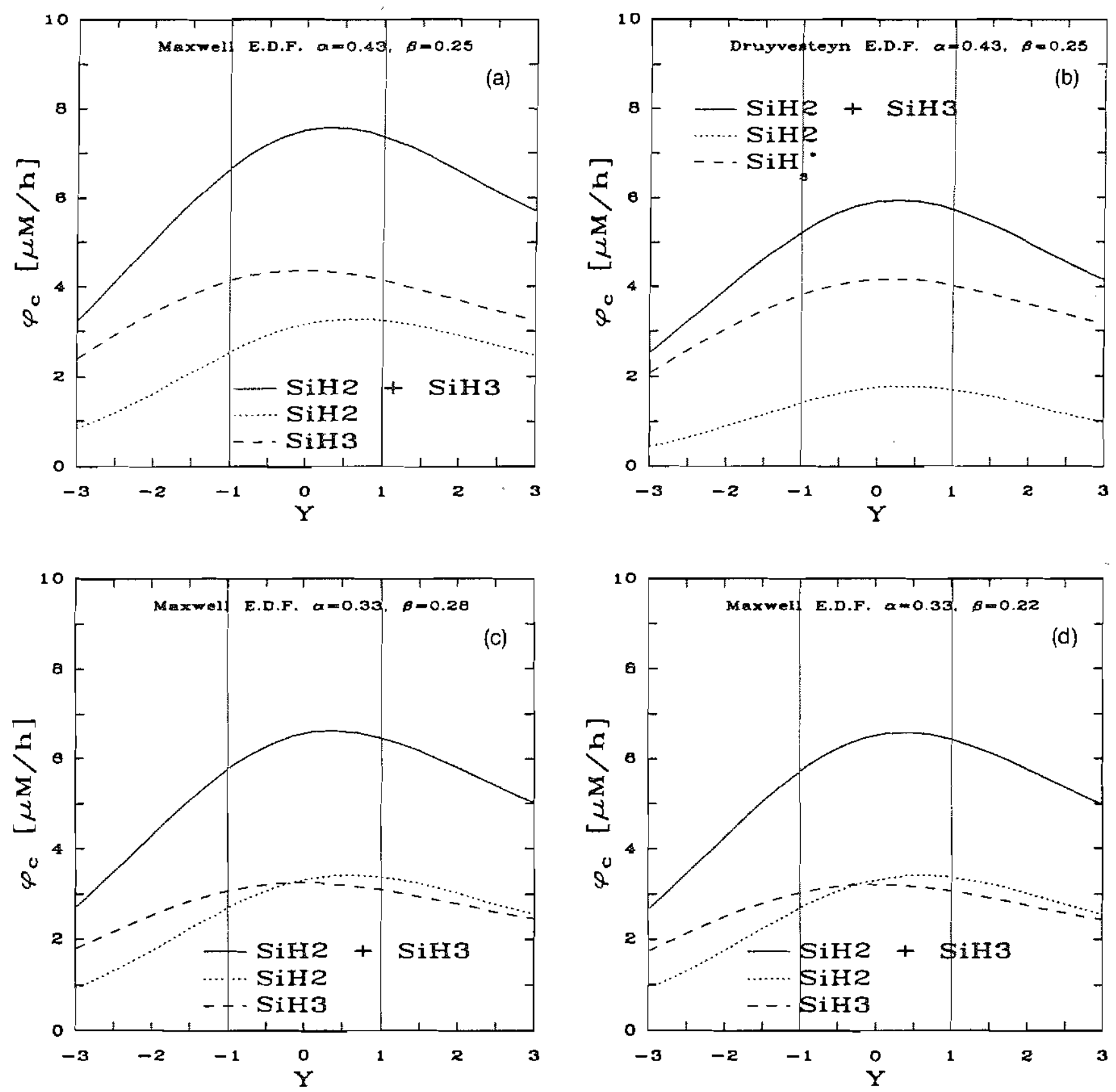

FIG. 13. Contribution of different radicais to total deposition ratc along the cathode for different electron distribution functions and different branching ratios. (a) Maxwell clectron distribution function; $\alpha=0.43$ and $\beta=0.25$. (b) Druyvesteyn electron energy distribution function; $\alpha=0.43$ and $\beta=0.25$. (c) Maxwell electron distribution function; $\alpha=0.33$ and $\beta=0.28$. (d) Maxwell electron distribution function; $\alpha=0.33$ and $\beta=0.22$.

\section{A. Glow discharge properties}

The Air discharge was considered in this study; i.e., the participation of $\mathrm{SiH}_{4}$ toward the formation of charged species was seen as negligible. According to the governing Eqs. (3)-(6) the following parameters determine the physical property of the discharge: (i) electron diffusion and mobility, $D_{c}$ and $\mu_{e}$; (ii) ion diffusion and mobility, $D_{p}$ and $\mu_{p}$; (iii) total inelastic and ionization collision efficiencies, $k$ and $a$; and (iv) secondary electron emission coefficient, $\gamma$.

The operation of the discharge covers the "rough vacuum' $0.1-10$ Torr and the temperature range 600$650 \mathrm{~K}$. Temperature and pressure dependencies of the parameters are reported where relevant, but the dependencies of mobilities and diffusion on the electric field were not considered. 


$$
\begin{aligned}
{ }^{d} n \frac{L 09 L}{d_{9} !^{\cdot} \mathscr{C} L Z} & = \\
\mathrm{I} & ={ }^{d d} n
\end{aligned}
$$

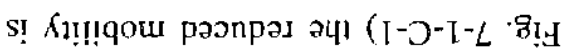

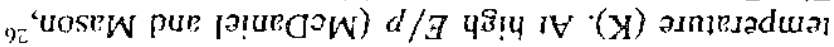

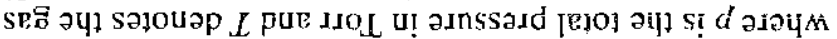

$$
\text { ( } \left.{ }_{\varepsilon-} \text { แง }\right) \quad \frac{L}{d}{ }_{81} 01 \times \angle S 9^{\circ} 6=N
$$

: K!!suep saqunu [e!O]

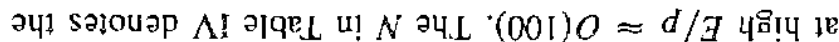

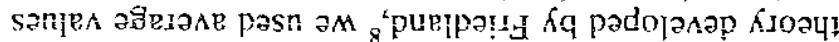

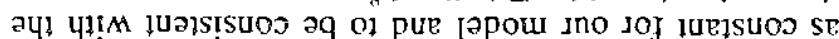

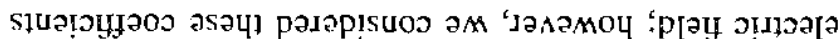

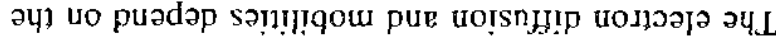

$\mathrm{I}[\tau / \varepsilon] s t$

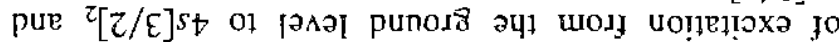

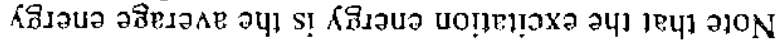
'Хर $009=L$ pur

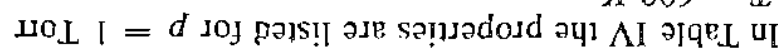

$\tau Z^{\circ} 0=g$ pue $E E O=0$

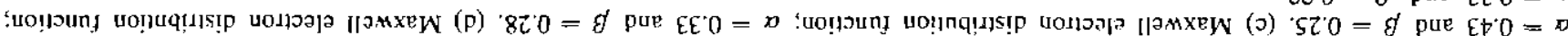

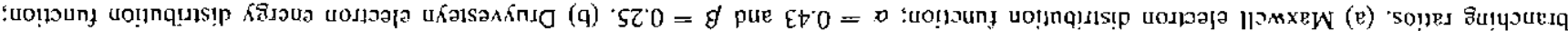

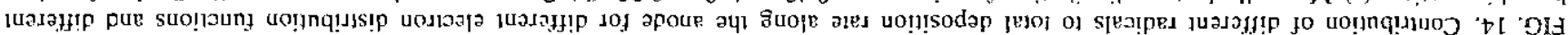
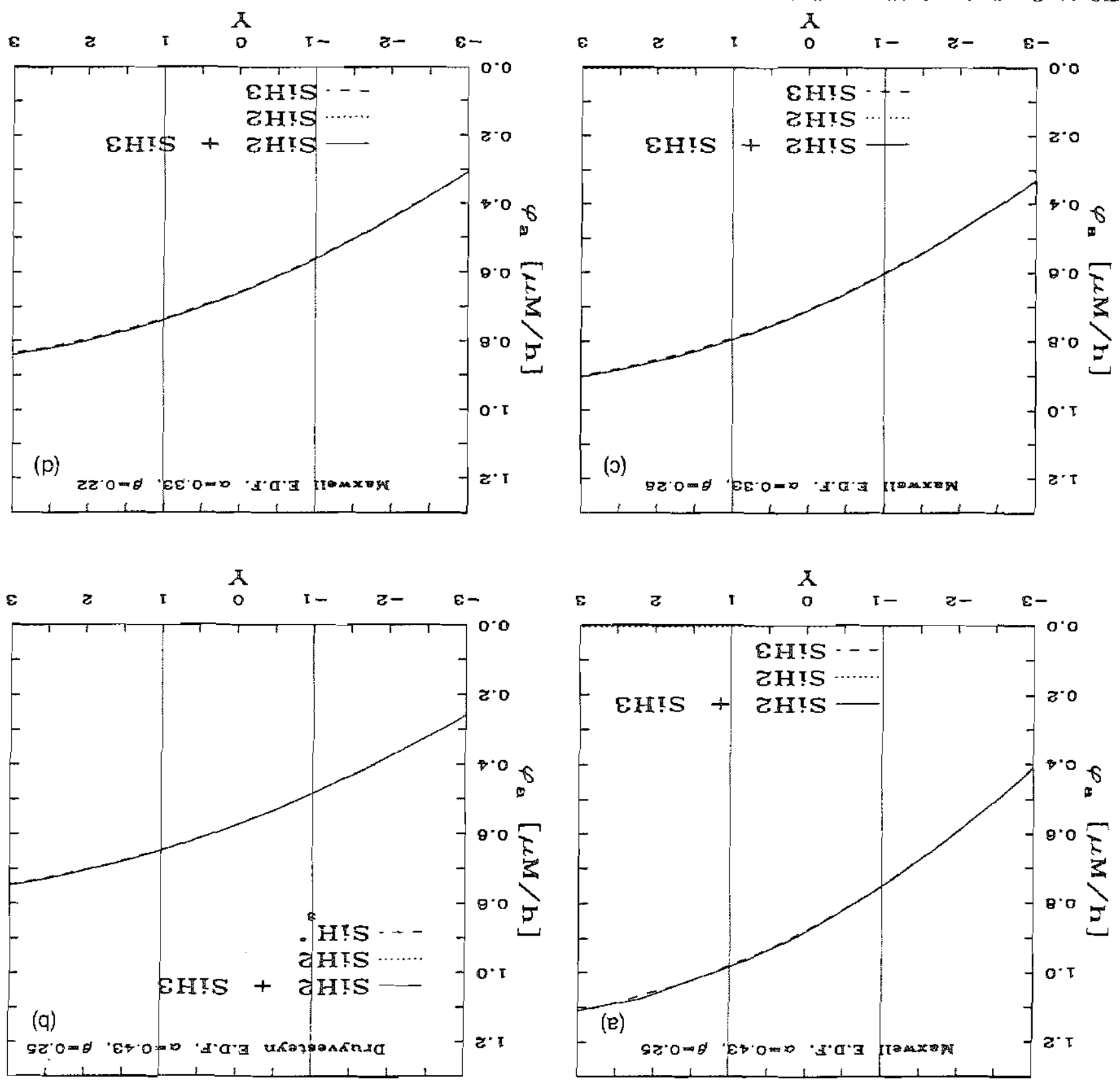
TABLE II. Parametric study of PECVD, cathode.

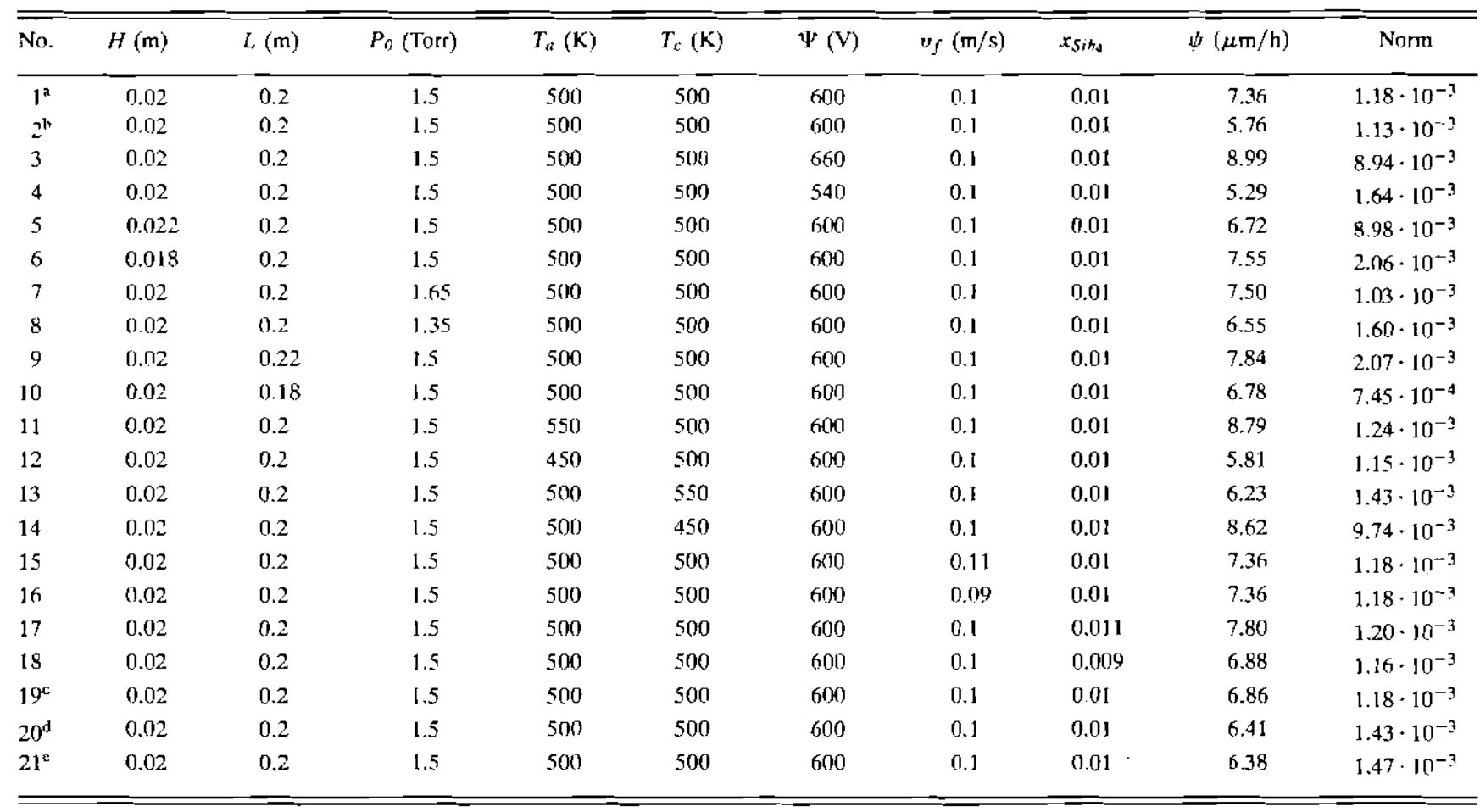

TABLE III. Parametric study of PECVD, anode.

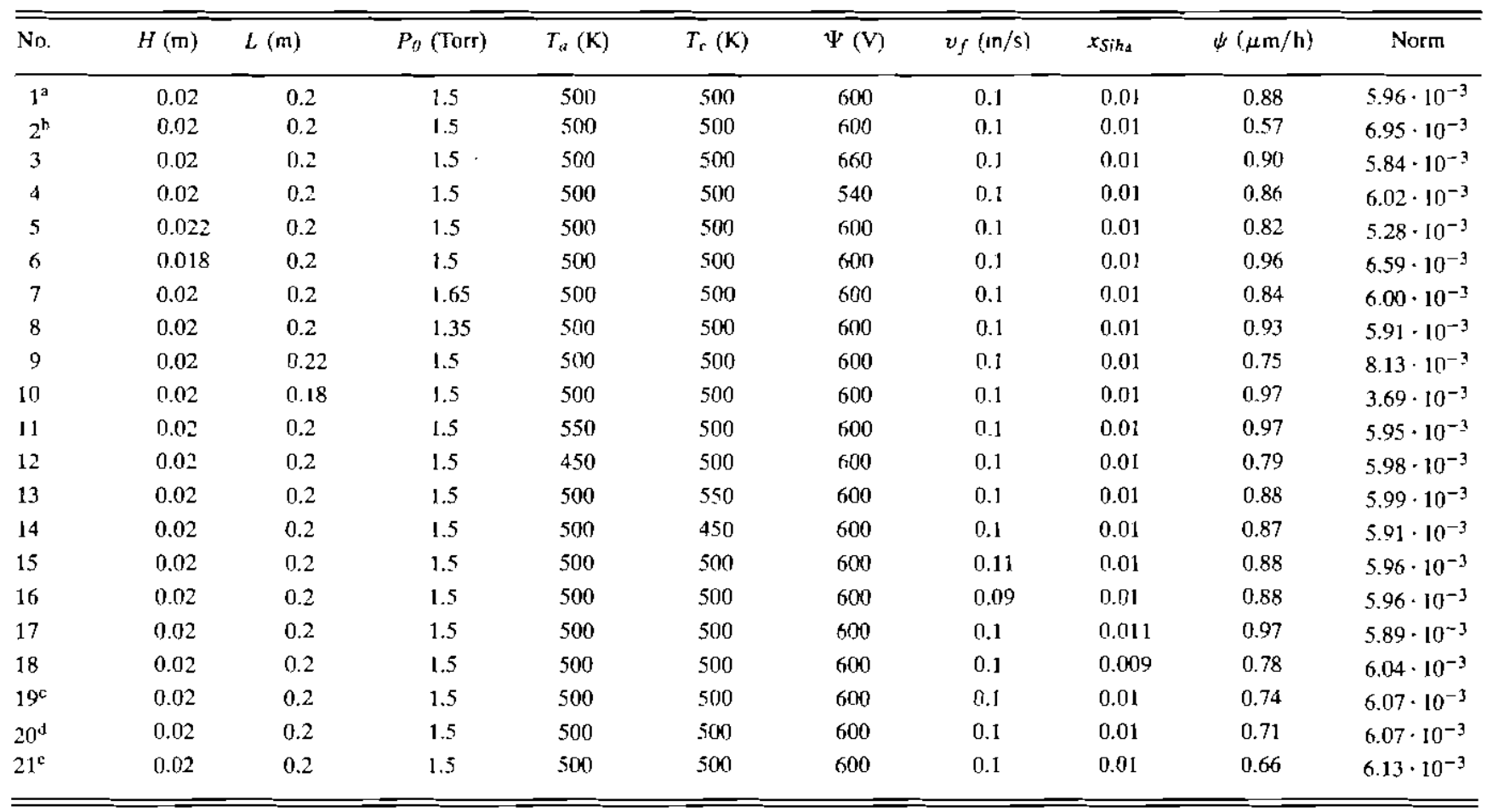

Reference case: Maxwell encrgy distribution; branching ratios: $\alpha=3 / 7$ and $\beta=12 / 49$.

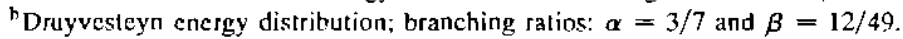

'Maxwell energy distritution; branching ratios: $\alpha=3 / 7$ and $\beta=4 / 21$.

"Maxwell encrgy distribulion; branching ratios: $\alpha=1 / 3$ and $\beta=2 / 7$.

${ }^{c}$ Maxwell energy distribution; branching tatios: $\alpha=1 / 3$ and $\beta=2 / 9$. 

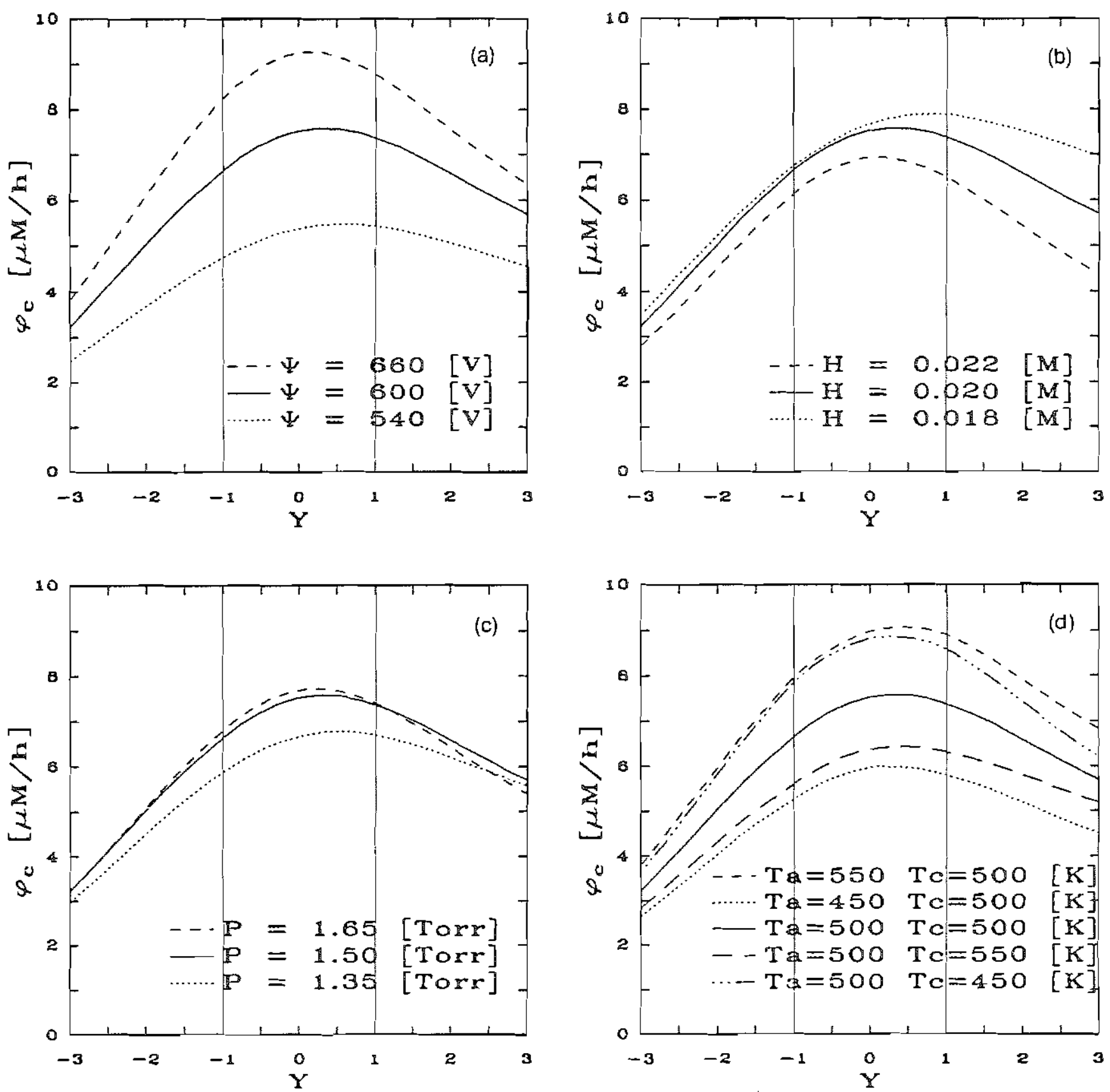

FIG. 15. Deposition rates along the cathode for different parameters. (a) Effect of ditferenl voltigcs, $V=660,600$, ind $540 \mathrm{~V}$. (b) Ellect of different gaps, $H=0.022,0.02$, and $0.018 \mathrm{~m}$. (c) Elfeet of different pressures, $P=1.65$, 1.5. and 1.35 Torr. (b) Ellect of different iemperalures on the anode and on the cathode; $T_{a}=550 \mathrm{~K}$ and $T_{c}=500 \mathrm{~K}, T_{a}=450 \mathrm{~K}$ and $T_{c}=500 \mathrm{~K}, T_{a}=500 \mathrm{~K}$ and $T_{c}=500 \mathrm{~K}, T_{a}=500 \mathrm{~K}$ and $T_{c}=550 \mathrm{~K}$, and $T_{a}=500 \mathrm{~K}$ and $T_{c}=450 \mathrm{~K}$

Hence,

$$
\mu_{p}=\frac{760 T}{273.16 p} \quad\left(\mathrm{~cm}^{2} \mathrm{~V}^{-1} \mathrm{~s}^{-1}\right)
$$

and the reduced mobility will be assumed to be constant for the range of operating conditions that are considered. The diffusion coefficient $D_{p}$ depends on gas temperature. The temperature dependence of the zero-field mobility $K_{0}$ for $\mathrm{Ar}^{+}$in Ar was reported in McDaniel and Mason ${ }^{26}$ (p. 311). We fitted a linear curve through their data and obtained

$$
K_{0}=-\frac{T}{450}+\frac{7}{3}
$$

The diffusion coefficient is then obtained from

$$
D_{p}=\frac{T^{2} K_{0}}{4172 p} \quad\left(\mathrm{~cm}^{2} \cdot \mathrm{s}^{-1}\right)
$$



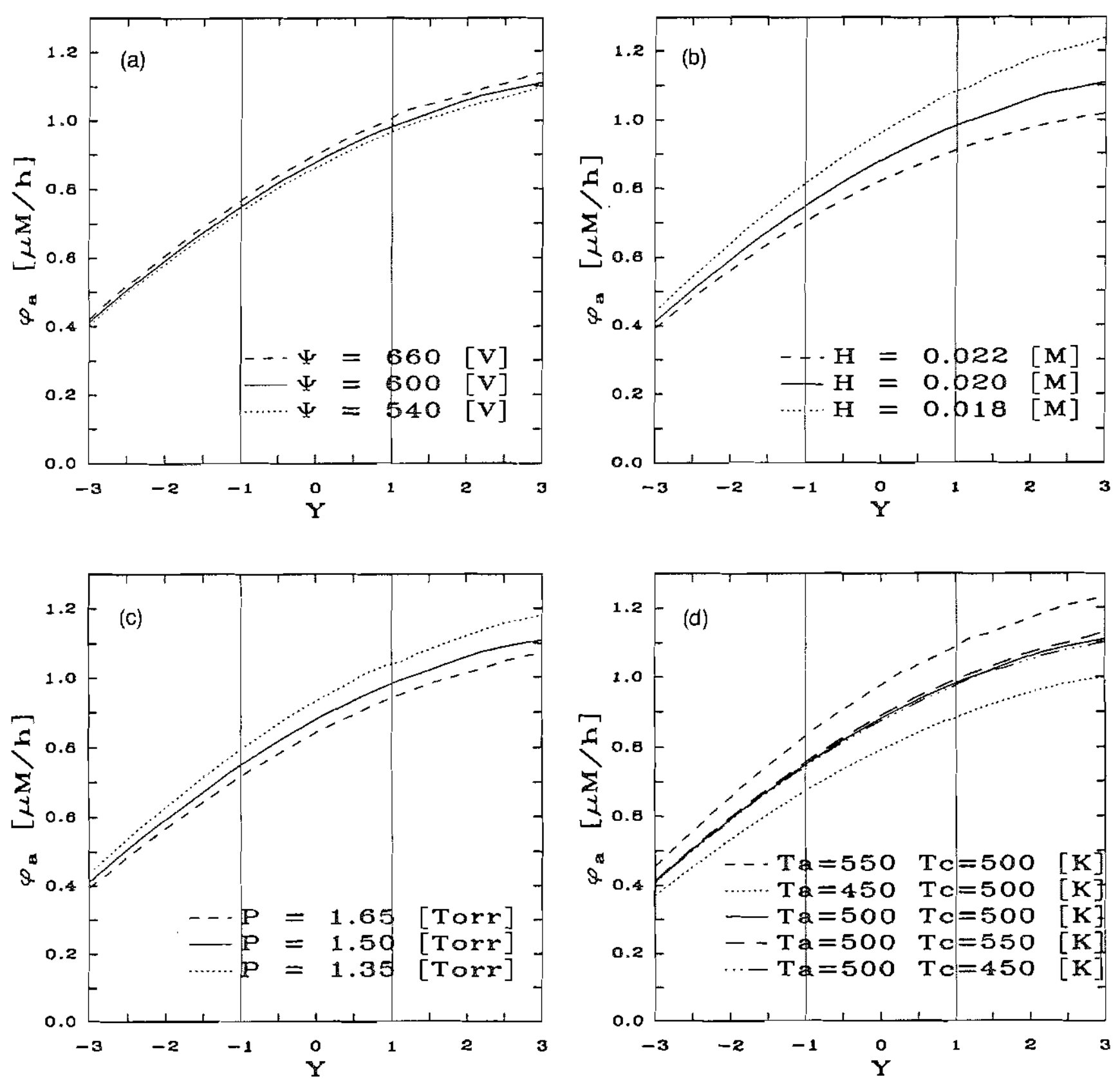

FIG. 16. Deposition rates along the anode for different parameters. (a) Effect of different voltages, $v=660,600$, and $540 \mathrm{~V}$. (b) Effect of different gaps, $H=0.022,0.02$, and $0.018 \mathrm{~m}$. (c) Effect of different pressures, $P=1.65,1.5$, and 1.35 Torr. (d) Effect of different temperatures on the anode and on the cathode; $T_{a}=550 \mathrm{~K}$ and $T_{c}=500 \mathrm{~K}, T_{a}=450 \mathrm{~K}$ and $T_{c}=500 \mathrm{~K}, T_{a}=500 \mathrm{~K}$ and $T_{c}=500 \mathrm{~K}, T_{a}=500 \mathrm{~K}$ and $T_{c}=550 \mathrm{~K}$, and $T_{a}=500 \mathrm{~K}$ and $T_{c}=450 \mathrm{~K}$.

These values can be compared to the values used by Park and Economou ${ }^{27}$ for an Ar-like plasma, which were reported at a gas temperature of $273 \mathrm{~K}$.

The total inelastic and ionization collision efficiencies are determined from the local-equilibrium form of the Friedland equation. If local-equilibrium holds, plots of $\alpha / p$ vs $(E / p)^{1 / 2}$ at large values of $E / p$ is linear with slope $a^{1 / 2}$ and abscissae $k \xi / 2$. Table IV of Kruithof and Penning, ${ }^{28}$ listing $\alpha / E$ for different $E / p$, was used to determine $a$ and $k$.

\section{B. Physical properties for hydrodynamic model}

Transport and physical properties of the $\mathrm{Ar} / \mathrm{SiH}_{4}$ mixture are required for the hydrodynamic model. To include the temperature dependence of the properties in the model, it is advantageous to use analytical expressions.

The Hirschfelder, Bird, Spotz formula was used to calculate the viscosities of the pure gases.

$$
\mu=2.2693 \times 10^{-6} \frac{(M T)^{1 / 2}}{\sigma^{2} \Omega^{2.2}} \quad \mathrm{~Pa} \cdot \mathrm{s}
$$


TABLE IV. Properties of glow distharges.

\begin{tabular}{|c|c|c|}
\hline Ptoperty & Value & Reterence \\
\hline$N D_{e}(E)$ & $3.3 \times 10^{22} \mathrm{~cm}^{-1} \cdot \mathrm{s}^{-1}$ & Suzuki et al, ${ }^{29}$ luh et al. \\
\hline$\frac{D_{e}}{\mu_{e}}(E)$ & $3.5 \mathrm{~V}$ & Suzuki et al. ${ }^{29}$ \\
\hline$D_{p}(p, T)$ & $86.29 \mathrm{~cm}^{2} \cdot \mathrm{s}^{-\mathrm{I}}$ & McDaniel and Mason 20 \\
\hline$\mu_{p}(p, T)$ & $1669.35 \mathrm{~cm}^{2} \cdot \mathrm{s}^{-1} \cdot \mathrm{v}^{-1}$ & McDanicl and Mason ${ }^{26}$ \\
\hline$a$ & $0.30(\mathrm{~V} \cdot \mathrm{cm} \cdot \text { Torr })^{-1}$ & Kruithof and Penning ${ }^{2 s}$ \\
\hline$k$ & $0.465\left(\mathrm{~V} \cdot \mathrm{cm} \cdot \mathrm{Torr}^{-1}\right.$ & Krnithof and Penning ${ }^{2 \gamma}$ \\
\hline $\begin{array}{l}\xi= \\
\frac{1}{2}\left(\xi_{i o n}+\xi_{e x}\right)\end{array}$ & $\frac{1}{2}(15.8+11.6) \mathrm{eV}$ & Carmint, ${ }^{31}$ Vicek ${ }^{10}$ \\
\hline$\gamma$ & 0.001 & Yamaguchi el al. ${ }^{23}$ \\
\hline
\end{tabular}

$M$ is the molecular weight, $T$ is the temperature, and $\sigma$ is a force constant defined in terms of the Lennard-Jones equation. $\Omega^{2,2}$ is a function of the variable $(k T / \epsilon)$. If experimental data were available, Sutherland's equation was used to present the temperature dependency of the gas viscosity

$$
\mu=B \frac{T^{3 / 2}}{T+C}
$$

where $B$ and $C$ are constants, characteristic for a given gas. To estimate the viscosity of a gaseous mixture, use was made of the Wilke equation

$$
\mu_{m}=\sum_{i}^{N} \frac{\mu_{i}}{\sum_{j}^{x_{i}} \phi_{i, j}}
$$

$\mu_{i}$ is the viscosity of the $i$-th component and $x_{i}$ is the mole fraction. $\phi_{i, j}$ is defined as follows:

$$
\phi_{i, j}=\frac{\left[1+\left(\frac{\mu_{i}}{\mu_{j}}\right)^{0.5}\left(\frac{M_{j}}{M_{i}}\right)^{0} \cdot 25\right]^{2}}{2^{1.5}\left(1+\frac{M_{i}}{M_{j}}\right)^{0.5}}
$$

Thermal conductivity estimates of the gases wete based on the kinetic theory for gases, taking molecular interactions into consideration. For a pure monatonic gas the Bird, Hirschfelder, Curtiss equation was used

$$
\lambda=8.3224 \times 10^{-2} \frac{(T / M)^{0.5}}{\sigma^{2} \Omega^{2,2}} \quad \mathrm{~W} \cdot \mathrm{m}^{-1} \cdot \mathrm{K}^{-1}
$$

For a pure polyatomic gas (in our case silane and radicals) the following relation was used:

$$
\lambda=2.6689 \times 10^{-3} \frac{(T / M)^{0.5}}{\sigma^{2} \Omega^{2,2}} \quad\left(C_{v}+18.702\right)
$$$$
W \cdot \mathrm{m}^{-1} \cdot \mathrm{K}^{-1}(70)
$$

The thermal conductivity of a gas mixfure was calculated by the Wassilica equation

$$
\lambda_{m}=\sum_{i}^{N} \frac{\lambda_{i}}{\sum j \frac{x_{j}}{x_{i}} A_{i, j}}
$$

The values of the coefficients $A_{i, j}$ can be calculated from the Lindsay and Bromley relationship

$$
\begin{aligned}
& A_{i, j}=0.25\left[1 .+\left(\frac{\mu_{i}}{\mu_{j}}\left(\frac{M_{j}}{M_{i}}\right)^{0.75} \frac{1+C_{i} / T}{1+C_{j} / T}\right)^{D .5}\right]^{2} \\
& \times \frac{1+C_{i j} / T}{1+C_{\mathrm{i}} / T}
\end{aligned}
$$

$C_{j}$ is the constant in the Sutherland equation for thermal conductivity. In addition, $C_{i j}=\left[C_{i} C_{j}\right]^{0.5}$.

Diffusion in gases is calculated from the Wilke and Lee equation

$$
D_{1,2}=\frac{B T^{1.5}}{p \sigma_{1,2}^{2} \Omega^{1,1}}\left(\frac{m_{1}+m_{2}}{m_{1} m_{2}}\right)^{0.5}
$$

and the coefficient $B$ is a function of the molecular weights of the gases

$$
B=2.14 \times 10^{-7}-4.92 \times 10^{-8}\left(\frac{M_{1}+M_{2}}{M_{1} M_{2}}\right)^{0.5}
$$

Thermal diffusion is calculated from the kinematic viscosity and the thermal diffusion ratio $K_{T D}$

$$
K_{T D}=5(C-1) \frac{S_{1} n_{1}-S_{2} n_{2}}{Q_{1} n_{1}^{2}+Q_{2} n_{2}^{2}+Q_{12} n_{1} n_{2}} n_{1} n_{2}
$$

where $n_{1}$ and $n_{2}$ are the volume fractions of the componcrits and

$$
\begin{aligned}
S_{1}= & m_{1} E_{1}-4 A m_{1} m_{2}-3 m_{2}\left(m_{2}-m_{1}\right) \\
Q_{1}= & E_{1}\left(6 m_{2}^{2}+(5-4 B) m_{1}^{2}+8 A m_{1} m_{2}\right) \\
Q_{12}= & 3\left(m_{1}-m_{2}\right)^{2}(5-4 B) \\
& +4 m_{1} m_{2} A(11-4 B)+2 E_{1} E_{2}
\end{aligned}
$$

with relations for $S_{2}$ and $Q_{2}$ derived as for $S_{1}$ and $Q_{1} \cdot m_{1}, m_{2}$, and $m$ are defincd as

$$
\begin{aligned}
m_{1} & =\frac{M_{1}}{M_{1}+M_{2}} \\
m_{2} & =\frac{M_{2}}{M_{1}+M_{2}} \\
m & =m_{1}-m_{2}
\end{aligned}
$$

$A, B, C, E_{1}$, and $E_{2}$ depend on the temperature and the interaction between molecules

$$
A=\frac{\Omega^{2,2}}{\Omega^{1.1}}
$$




$$
\begin{aligned}
B & =\frac{5 \Omega^{1,2} \Omega^{1,3}}{5 \Omega^{1,1}} \\
C & =\frac{2 \Omega^{1,2}}{5 \Omega^{1,1}} \\
E_{1} & =\frac{2 \Omega^{2,2}}{5(1-m) \Omega^{1,1}} \\
E_{2} & =\frac{2 \Omega^{2,2}}{5(1+m) \Omega^{1,1}}
\end{aligned}
$$

All these relations were included in a program to calculate the properties of any ratio between $\mathrm{Ar}$ and $\mathrm{SiH}_{4}$ at any pressure and temperature. The extension to include different gaseous components, e.g., $\mathrm{N}_{2}$ or $\mathrm{Cl}_{2}$, is straightforward. 